\title{
T duality and Wald entropy formula in the Heterotic Superstring effective action at first-order in $\alpha^{\prime}$
}

\author{
Zachary Elgood and Tomás Ortín \\ Instituto de Física Teórica UAM/CSIC, \\ C/ Nicolás Cabrera, 13-15, C.U. Cantoblanco, Madrid E-28049, Spain \\ E-mail: zachary.elgood@uam.es, tomas.ortin@csic.es
}

ABSTRACT: We consider the compactification on a circle of the Heterotic Superstring effective action to first order in the Regge slope parameter $\alpha^{\prime}$ and re-derive the $\alpha^{\prime}$-corrected Buscher rules first found in ref. [42], proving the T duality invariance of the dimensionallyreduced action to that order in $\alpha^{\prime}$. We use Iyer and Wald's prescription to derive an entropy formula that can be applied to black-hole solutions which can be obtained by a single non-trivial compactification on a circle and discuss its invariance under the $\alpha^{\prime}$-corrected $\mathrm{T}$ duality transformations. This formula has been successfully applied to $\alpha^{\prime}$-corrected 4 dimensional non-extremal Reissner-Nordström black holes in ref. [21] and we apply it here to a heterotic version of the Strominger-Vafa 5-dimensional extremal black hole.

Keywords: Black Holes in String Theory, String Duality, Superstrings and Heterotic Strings

ARXIV EPRINT: 2005.11272 


\section{Contents}

1 Introduction 1

2 The Heterotic Superstring effective action to $\mathcal{O}\left(\alpha^{\prime}\right) \quad 6$

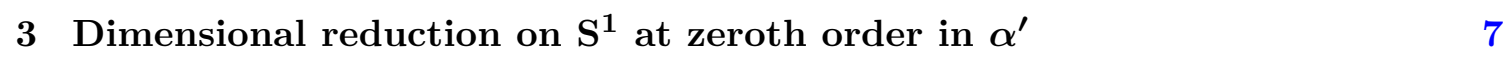

4 Dimensional reduction on $\mathrm{S}^{\mathbf{1}}$ at $\mathcal{O}\left(\alpha^{\prime}\right) \quad 9$

$\begin{array}{lll}4.1 \text { T duality } & 15\end{array}$

$\begin{array}{lll}5 & \text { Entropy formula } & \mathbf{1 7}\end{array}$

5.1 The Wald entropy of the $\alpha^{\prime}$-corrected Strominger-Vafa black hole $\quad 17$

6 Discussion 20

A Relation between 10- and 9-dimensional fields at zeroth order in $\alpha^{\prime} \quad 21$

B Relation between 10- and 9-dimensional fields at $\mathcal{O}\left(\alpha^{\prime}\right) \quad 22$

\section{Introduction}

Superstring Theory is expected to be a consistent theory of Quantum Gravity. Therefore, one would like to use it to study gravitational systems in which quantum-mechanical effects are believed to play an important role, such as black holes. In particular, one of the results that we expect from Superstring Theory is a microscopical accounting of the entropy attributed to them by macroscopic (thermodynamic) laws and calculations.

Achieving this result demands, first of all, black-hole solutions of Superstring Theory whose macroscopic entropy can be computed. These are classical solutions of the Superstring effective action. Then, if one manages to associate the black-hole solution to a good Superstring Theory background on which the theory can be quantized, the microscopic entropy can be associated to the density of string states in that background.

In a seminal paper, [1] Strominger and Vafa completed the above program for a extremal, static, 3-charge 5-dimensional black-hole solution of the type IIB Superstring Theory at lowest order in the Regge slope parameter $\alpha^{\prime}$, identifying the associated type IIB string background as one with intersecting D1- and D5-branes with momentum flowing along the intersection. Strominger and Vafa argued that, although the black hole only solved the zeroth-order in $\alpha^{\prime}$ equations of motion, the higher-order corrections could be made small enough by imposing conditions on the charges carried by the black hole. Under those conditions, the microscopic and macroscopic entropies (the later given simply by the one fourth of the area of the event horizon) matched to lowest order in $\alpha^{\prime}$.

Since $\alpha^{\prime}$ is the square of the string length, the higher-order in $\alpha^{\prime}$ corrections to the string effective action, its solutions and the properties of the solutions describe characteristic "stringy" deviations and this makes their study most interesting. This study requires: 
1. The knowledge of the higher-order terms in the string effective field-theory actions.

2. The construction of solutions of those effective actions with higher-order terms. These solutions can often be viewed as $\alpha^{\prime}$-corrected zeroth-order solutions (recovered by setting $\left.\alpha^{\prime}=0\right)$.

3. The computation of the physical properties of the $\alpha^{\prime}$-corrected solutions.

Terms of higher-order in $\alpha^{\prime}$ are terms of higher order in curvatures and their complexity grows rapidly with the power of $\alpha^{\prime}$. This makes them very difficult to compute and, consequently, our knowledge of the $\alpha^{\prime}$ corrections to the effective field theory actions of different Superstring Theories is very limited. The $\alpha^{\prime}$ corrections to the Heterotic Superstring effective action are probably the best known, and they have only been computed to cubic order (quartic in curvatures) in ref. [2], using supersymmetry completion of the Lorentz Chern-Simons terms [3]. ${ }^{1}$

We can use, then, the Heterotic Superstring effective action given in ref. [2] for the next step: computing $\alpha^{\prime}$ corrections to black-hole solutions. As a matter of fact, the black-hole solution studied by Strominger and Vafa in ref. [1] can also be considered as a zeroth-order solution of the Heterotic Superstring effective action and it would certainly be interesting to compute its $\alpha^{\prime}$ corrections, at least to first order. Finding these corrections, though, is a complicated problem. One of the problems is that the complete Heterotic Superstring effective action with higher-order corrections has not been compactified down to the 5 dimensions in which the black hole lives. ${ }^{2}$ Effective actions which would capture what are believed to be the most relevant $\alpha^{\prime}$ corrections in lower dimensions have been proposed and used to compute corrections to black-hole solutions (see, e.g. ref. [12] and references therein). Alternatively, in order to simplify the problem, it has been proposed to work only with the near-horizon solution (see e.g. refs. [13, 14] and references therein and more recent work in the Type IIA compactified on K3 setup $[15,16])$. It is fair to say that each of these simplified approaches has problems of its own and that they do not offer a complete picture of what the $\alpha^{\prime}$-corrected black-hole solutions are like.

Recently, a different approach for computing $\alpha^{\prime}$ corrections without making assumptions about the lower-dimensional effective actions or considering only near-horizon limits has been proposed in ref. [17]: since the 10-dimensional first-order in $\alpha^{\prime}$ Heterotic Superstring effective action is known without any ambiguities (beyond possible field redefinitions), first-order in $\alpha^{\prime}$ corrections to solutions should be directly computed in 10 dimensions using the uplift of 4- or 5-dimensional solutions. Then, the $\alpha^{\prime}$-corrected solutions can be compactified back to 4 - or 5-dimensions. This approach has been successfully used

\footnotetext{
${ }^{1}$ The equivalence of this effective action with previous results obtained in refs. [4-7] was established in ref. [8].

${ }^{2} \mathrm{~A}$ toroidal compactification to first order in $\alpha^{\prime}$ but with no Yang-Mills fields has been recently constructed in ref. [9]. The toroidal compactification with only Abelian Yang-Mills fields (which occur at first order in $\alpha^{\prime}$ ) and no terms involving the torsionful spin connection (so the 10-dimensional action is that of $\mathcal{N}=1, d=10$ supergravity coupled to Abelian vector supermultiplets) was carried out in [10]. An earlier compactification of the Heterotic Superstring effective action to just $d=4$ at zeroth-order in $\alpha^{\prime}$ (so the 10-dimensional action is that of pure $\mathcal{N}=1, d=10$ supergravity) was carried out in [11].
} 
to compute the first-order in $\alpha^{\prime}$ corrections to 5- and 4-dimensional extremal black holes in refs. [17] and [18-20], respectively and, more recently, to 4-dimensional non-extremal Reissner-Nordström black holes in ref. [21]. The question of the regularity of the so-called small black holes has also been reviewed in refs. $[22,23]$ in the light of those results.

Having the $\alpha^{\prime}$-corrected solutions we can compute their physical properties. For black holes, these are their conserved charges and their thermodynamical properties: entropy and temperature. The Hawking temperature is always determined by the value of the surface gravity of the metric. While the metric can receive $\alpha^{\prime}$ corrections, the relation between Hawking temperature and surface gravity does not change. This is not the case for the Bekenstein-Hawking entropy, which, in presence of $\alpha^{\prime}$ corrections (higher-order in curvature corrections in general) is no longer determined by the area of the horizon which also receives $\alpha^{\prime}$ corrections coming from those of the metric. Based on previous work [24, 25], in ref. [26] Iyer and Wald gave a prescription to derive an entropy formula in diffeomorphism-invariant theories. The main fact that characterizes this prescription is that the entropy computed using it satisfies the first law of black-hole mechanics [27].

Iyer and Wald's prescription is based on a series of assumptions about the field content, which has to consists of tensor fields only. The only tensor field in our current understanding of Nature is the metric, the rest being connections and sections of different gauge bundles or, in other words, field with some kind of gauge freedom. The validity of Iyer and Wald's prescription has subsequently extended to theories that include fields with gauge freedoms in refs. [28-30], but the Heterotic Superstring effective action (and many other string effective actions) include a field which is not a connection or a section of some gauge bundle: the Kalb-Ramond field. This complication has been ignored in most of the string literature $^{3}$ and the Iyer-Wald prescription has been naively applied with results that seem to be compatible with the microscopic calculations of the entropy. ${ }^{4}$

For instance, in ref. [17], the entropy of the (heterotic version of the) $\alpha^{\prime}$-corrected Strominger-Vafa black hole was computed using the Iyer-Wald prescription directly in the 10-dimensional action. The result obtained was compatible with that of the microscopic calculation carried out in ref. [32] to first-order in $\alpha^{\prime}$, with an appropriate identification between the charges carried by the black hole and associated string background [33]. More precisely, the entropy obtained was interpreted in ref. [17] as the $\mathcal{O}\left(\alpha^{\prime}\right)$ truncation of the expansion in powers of $\alpha^{\prime}$ of the exact result found in ref. [32].

\footnotetext{
${ }^{3}$ An independent derivation of an entropy formula using Wald's formalism and dealing with some of the problems that the presence of the Kalb-Ramond field raises has been made in ref. [31]. The final entropy formula derived there depends on a compensating gauge parameter which was left undetermined. This makes a comparison with the entropy formula we will derive impossible. For instance, it is not possible to compute the entropy of the Strominger-Vafa black hole using this formula, unless one can prove that the unknown term does not contribute to it. Although in that reference it is argued that, at least in certain relevant cases, this is indeed the case. In the same reference it is also shown that the invariance of their entropy formula under local Lorentz transformations depends on it, which seems contradictory.

${ }^{4}$ Wald's formalism's first step consists in the proof of a first law of black-hole mechanics for the theory under consideration. A first law for the Heterotic Superstring effective action to first order in $\alpha^{\prime}$ has not yet been proven, although it is widely assumed to exist (for instance, in the derivation of the entropy formula of ref. [31]).
} 
This interpretation, however, was a bit puzzling, because in ref. [17], it was argued that the near-horizon region of the black-hole solution, which determines the entropy, should not receive further $\alpha^{\prime}$ corrections. ${ }^{5}$ Furthermore, an explicit calculation shows that at least the $\mathcal{O}\left(\alpha^{\prime 2}\right)$ corrections to the entropy vanish identically [34]. All this suggests that the result obtained for the entropy in ref. [17] should be exact to all orders in $\alpha^{\prime}$ and, therefore, it should be identical to the result of the microscopic calculation of ref. [32].

This puzzle was solved in ref. [33], where it was observed that the dependence of the action on the Riemann curvature $^{6}$ in the Lorentz Chern-Simons term of the KalbRamond field strength is changed by dimensional reduction. Taking into account this change, which amounts to a factor of 2 with respect to the result of ref. [17], the macroscopic entropy computed at first order in $\alpha^{\prime}$ using naively the Iyer-Wald formula matches the exact microscopic result. This gives further support to the conjecture that the black-hole solution does not receive further $\alpha^{\prime}$ corrections and may be considered an exact Heterotic Superstring solution.

The results of ref. [33] made clear that, in the case of the Heterotic Superstring effective action, the entropy formula has to be derived from the dimensionally-reduced action in order to determine correctly the dependence of the action of the lower-dimensional Riemann tensor. One of our goals in this paper is to perform the dimensional reduction of the Heterotic Superstring effective action to first order in $\alpha^{\prime}$ over a circle to apply to it the Iyer-Wald prescription and obtain an entropy formula. This entropy formula can only be applied to $d$-dimensional black holes that can be obtained by trivial compactification on $\mathrm{T}^{9-d}$ and a non-trivial compactification on a circle. For instance, it can be applied to the heterotic version of the Strominger-Vafa black hole because it can be obtained from a 10-dimensional solution by trivial compactification on $\mathrm{T}^{4}$, to 6 dimensions and a nontrivial compactification on a circle from 6 to 5 dimensions. It can also be applied to the non-supersymmetric 4-dimensional Reissner-Nordström black hole of ref. [35], which can be obtained from pure 5-dimensional gravity and, therefore, can be obtained from a purely gravitational 10-dimensional solution by trivial compactification on $\mathrm{T}^{5}$ to 5 dimensions and, then, by a non-trivial compactification on a circle from 5 to 4 dimensions. Actually, the entropy formula eq. (5.2b) that we are going to derive in section 5 has been applied to a non-extremal version of the 4-dimensional Reissner-Nordström black hole we just discussed, in ref. [21]. While the microscopic interpretation of the entropy of this black hole is unknown, being a black hole with finite temperature, one can check that the first law of thermodynamics is indeed satisfied because the temperature computed from the $\alpha^{\prime}$-corrected metric and the entropy computed from the $\alpha^{\prime}$-corrected metric with the $\alpha^{\prime}$ corrected entropy formula are related by the thermodynamic relation

$$
\frac{\partial S}{\partial M}=\frac{1}{T} \text {. }
$$

This paper's second goal has to do with one of the most interesting and characteristic properties of String Theory: T duality. ${ }^{7}$ T duality relates two string theories compactified

\footnotetext{
${ }^{5}$ The complete black-hole solution may receive further corrections.

${ }^{6}$ According to the Iyer-Wald prescription, the entropy formula only depends on the occurrences of the Riemann tensor in the action.

${ }^{7}$ For a review with many early references see ref. [36].
} 
in circles of dual radii. The spectra of the two theories can be put into one-to-one correspondence and, from the lower dimensional point of view, they are essentially identical, up to charge identifications. ${ }^{8}$ More generally, Buscher [37, 38] showed that two string backgrounds with one isometry whose background fields are related by the so-called Buscher $T$ duality rules are equivalent.

String backgrounds related by $\mathrm{T}$ duality may have very different geometries and properties in spite of their stringy equivalence. On the other hand, T duality (via the Buscher rules) can be used actively to generate new (dual) string backgrounds from known backgrounds. This is what makes this duality so interesting.

Perhaps not surprisingly, the Buscher rules can be derived from the string effective action: the dual ${ }^{9}$ Kaluza-Klein compactifications of two effective actions on a circle give the same $(d-1)$-dimensional action and the same equations of motion. In practice, one can perform identical Kaluza-Klein compactifications, determine the relation between the $(d-1)$-dimensional fields of the two actions (which is usually very simple because it does not involve the $(d-1)$-dimensional string metric or Kalb-Ramond field) and rewrite this relation in terms of the components of the original $d$-dimensional fields [39]. This relation is just the Buscher $\mathrm{T}$ duality rules. This strategy has been successfully used to find the extension of the Buscher $\mathrm{T}$ duality rules that relates equivalent type IIA and type IIB superstring backgrounds [40] and higher-rank Ramond-Ramond potentials [41].

In the context of the Heterotic Superstring, this strategy was used in [42] to find the first-order in $\alpha^{\prime}$ corrections to the Buscher rules. ${ }^{10}$ Only the Yang-Mills fields were included at order $\alpha^{\prime}$, but, taking into account that the torsionful spin connection enters the action in exactly the same way as the Yang-Mills fields [3], it was possible to find the $\alpha^{\prime}$ corrections to the Buscher rules.

The $\alpha^{\prime}$-corrected Buscher rules are of no use if there are no $\alpha^{\prime}$-corrected solutions at one's disposal to generate new solutions or to check their equivalence. For this reason, the results of ref. [42] were sleeping the "sleep of the just" 11 until quite recently, when they were first applied to $\alpha^{\prime}$-corrected self-T-dual solutions, providing a highly non-trivial test of both the $\alpha^{\prime}$ corrections of the solutions and of the T duality rules.

Our second goal will be to study the T duality invariance of the complete dimensionallyreduced Heterotic Superstring effective action and of the entropy formula that follows from it. While the $\alpha^{\prime}$-corrected Buscher rules will be those of ref. [42], the complete reduced action will have many more $\mathcal{O}\left(\alpha^{\prime}\right)$ terms than the action obtained there. The invariance of the action under $\mathrm{T}$ duality suggests that they will contribute to the entropy in a $\mathrm{T}$ dualityinvariant form, and we will prove that this is the case. ${ }^{12}$

\footnotetext{
${ }^{8}$ Charges related to Kaluza-Klein momentum and charges related to the winding number along the compact direction should be interchanged.

${ }^{9}$ That is, with fields related by the Buscher rules.

${ }^{10}$ At zeroth-order in $\alpha^{\prime}$, the Heterotic Superstring effective action only describes the so-called common sector of Neveu-Schwarz-Neveu-Schwarz fields, so the Buscher rules are just those found by Buscher.

${ }^{11}$ As a matter of fact, they have partially re-derived several times [43, 44]. Other studies of the effect of $\alpha^{\prime}$ corrections on $\mathrm{T}$ duality and $\mathrm{O}(d, d)$ transformations in toroidal compactifications, sometimes in extended set-ups (such as Double Field Theory) can be found [9, 45-49].

${ }^{12}$ It follows trivially from the invariance of the lower-dimensional string metric and dilaton under $\mathrm{T}$ duality that the zeroth-order in $\alpha^{\prime}$ temperature and entropy (the area) are also $\mathrm{T}$ duality invariant. This property
} 
This paper is organized as follows: we introduce the Heterotic Superstring effective action to first order in $\alpha^{\prime}$ following ref. [2] in section 2. In section 3, we revisit the dimensional reduction on a circle of the action at zeroth order in $\alpha^{\prime}$ as a warm-up exercise and also because we will need some of the results when we consider the higher-order terms in section 4 . In that section we will obtain the complete dimensionally-reduced action to first order in $\alpha^{\prime}$, we will find the $\mathrm{T}$ duality rules and we will prove the invariance of the action under those $\mathrm{T}$ duality rules. In section 5 , we will use the dimensionally-reduced $\mathrm{T}$ dualityinvariant action to derive an entropy formula using the Iyer-Wald prescription and we will apply it to the heterotic version of the $\alpha^{\prime}$-corrected Strominger-Vafa black hole of ref. [17]. We will end by discussing our results and future work on these topics in section 6 .

\section{The Heterotic Superstring effective action to $\mathcal{O}\left(\alpha^{\prime}\right)$}

Let us start by reviewing the Heterotic Superstring effective action to $\mathcal{O}\left(\alpha^{\prime}\right)$. We will use the formulation given in ref. [2], but written in the conventions of ref. [52]. ${ }^{13}$ In this formulation, the action is constructed recursively order by order in $\alpha^{\prime}$.

The zeroth-order 3 -form field strength of the Kalb-Ramond 2-form $B$ is defined as

$$
H_{\mu \nu \rho}^{(0)} \equiv 3 \partial_{[\mu} B_{\nu \rho]}
$$

and it contributes as torsion to the zeroth-order torsionful spin connections

$$
\Omega_{( \pm) \mu b}^{(0){ }^{a} b}=\omega_{\mu b}^{a} \pm \frac{1}{2} H_{\mu b}^{(0)}{ }_{\mu}^{a},
$$

where $\omega_{\mu}^{a} b$ is the (torsionless, metric-compatible) Levi-Civita spin connection 1-form.

The corresponding zeroth-order Lorentz curvature 2-forms and Chern-Simons 3-forms are defined as

$$
\begin{aligned}
& R_{( \pm) \mu \nu b}^{(0)}{ }^{a}=2 \partial_{[\mu \mid} \Omega_{( \pm) \mid \nu] b}^{(0)}{ }^{a}-2 \Omega_{( \pm)[\mu \mid c}^{(0)}{ }^{a} \Omega_{( \pm) \mid \nu]}^{(0)} b, \\
& \omega_{( \pm)}^{\mathrm{L}(0)}=3 R_{( \pm)[\mu \nu \mid}^{(0)}{ }_{b} \Omega_{( \pm) \mid \rho]}^{(0)}{ }^{b}{ }_{a}+2 \Omega_{( \pm)[\mu \mid}^{(0)}{ }_{b}{ }^{(0)} \Omega_{( \pm)|\nu|}^{(0)}{ }^{b} \Omega_{( \pm) \mid \rho]}^{(0)}{ }^{c} a .
\end{aligned}
$$

The gauge field 1-form is $A_{\mu}^{A}$, where $A, B, C, \ldots$ are the adjoint gauge indices of some group that we will not specify. The gauge field strength and the Chern-Simons 3-forms are defined by

$$
\begin{aligned}
F_{\mu \nu}^{A} & =2 \partial_{[\mu} A_{\nu]}^{A}+f_{B C} A^{A} A_{[\mu}^{B} A_{\nu]}^{C}, \\
\omega^{\mathrm{YM}} & =3 F_{A[\mu \nu} A_{\rho]}^{A}-f_{A B C} A^{A}{ }_{[\mu} A^{B}{ }_{\nu} A^{C}{ }_{\rho]},
\end{aligned}
$$

was proven by Horowitz and Welch in ref. [50] before the relation between the Buscher rules and dimensional reduction was established in ref. [39]. Recently, it has been investigated again from the same point of view in refs. [31, 51] to first order in $\alpha^{\prime}$, but, again, the relation between dimensional reduction and $\mathrm{T}$ duality and the invariance of the lower-dimensional string metric and dilaton field lead, trivially, to the invariance of the $\alpha^{\prime}$-corrected temperature. The invariance of the action under $\mathrm{T}$ duality at this order implies that of the entropy formula using the Iyer-Wald prescription because the Riemann curvature is $\mathrm{T}$ duality invariant.

${ }^{13}$ The relation with the fields in ref. [2] can be found in ref. [53]. 
where we have lowered the adjoint group indices using the Killing metric of $K_{A B}: f_{A B C} \equiv$ $f_{A B}{ }^{D} K_{D C}$ and of the gauge fields $F_{A \mu \nu} \equiv K_{A B} F^{B}{ }_{\mu \nu}$.

Then, at first order

$$
\begin{aligned}
& H_{\mu \nu \rho}^{(1)}=3 \partial_{[\mu} B_{\nu \rho]}+\frac{\alpha^{\prime}}{4}\left(\omega_{\mu \nu \rho}^{\mathrm{YM}}+\omega_{(-) \mu \nu \rho}^{\mathrm{L}(0)}\right), \\
& \Omega_{( \pm) \mu^{a} b}^{(1){ }^{a}}=\omega_{\mu b}^{a} \pm \frac{1}{2} H_{\mu}^{(1) a}{ }_{b}, \\
& R_{( \pm) \mu \nu b}^{(1) \quad a}=2 \partial_{[\mu \mid} \Omega_{( \pm) \mid \nu] b}^{(1)} a_{b}-2 \Omega_{( \pm)\left[\mu \mid c{ }^{a}{ }^{(1)} \Omega_{( \pm) \mid \nu]}^{(1)}{ }^{c} b\right.}, \\
& \omega_{( \pm) \mu \nu \rho}^{\mathrm{L}(1)}=3 R_{( \pm)[\mu \nu \mid b}^{(1)}{ }^{a} \Omega_{( \pm) \mid \rho]^{b}}^{(1)}+2 \Omega_{( \pm)[\mu \mid}^{(1)}{ }^{a} \Omega_{( \pm)|\nu|}^{(1)}{ }^{b} \Omega_{( \pm) \mid \rho]}^{(1)}{ }^{c} . \\
& H_{\mu \nu \rho}^{(2)}=3 \partial_{[\mu} B_{\nu \rho]}+\frac{\alpha^{\prime}}{4}\left(\omega^{\mathrm{YM}}{ }_{\mu \nu \rho}+\omega_{(-) \mu \nu \rho}^{\mathrm{L}(1)}\right) \text {, }
\end{aligned}
$$

etc.

Only $\Omega_{( \pm) \mu}^{(0)}, R_{( \pm) \mu \nu}^{(0)}{ }^{a}, \omega_{( \pm) \mu \nu \rho}^{\mathrm{L}(0)}$ and $H_{\mu \nu \rho}^{(1)}$ (plus the Yang-Mills fields) occur in the action. In practice, though, it is more convenient to work with the higher-order objects, neglecting the terms of higher order in $\alpha^{\prime}$ when necessary. Thus, from now on we will suppress the $(n)$ upper indices when they do not play a relevant role.

In terms of all these objects, the Heterotic Superstring effective action in the string frame and to first-order in $\alpha^{\prime}$ can be written as

$$
S=\frac{g_{s}^{2}}{16 \pi G_{N}^{(10)}} \int d^{10} x \sqrt{|g|} e^{-2 \phi}\left\{R-4(\partial \phi)^{2}+\frac{1}{12} H^{2}-\frac{\alpha^{\prime}}{8}\left[F_{A} \cdot F^{A}+R_{(-)}{ }^{a} b \cdot R_{(-)}{ }^{b} a\right]\right\},
$$

where $G_{N}^{(10)}$ is the 10-dimensional Newton constant, $\phi$ is the dilaton field, the vacuum expectation value of $e^{\phi}$ is the Heterotic Superstring coupling constant $g_{s}, R$ is the Ricci scalar of the string-frame metric $g_{\mu \nu}$ and the dot indicates the contraction of the indices of 2-forms: $F_{A} \cdot F^{A} \equiv F_{A \mu \nu} F^{A \mu \nu}$.

\section{Dimensional reduction on $\mathrm{S}^{1}$ at zeroth order in $\alpha^{\prime}$}

As a warm-up exercise (and also because of the recursive definition of the action that will make necessary the zeroth-order fields in the first-order action), we review the wellknown dimensional reduction of the action at zeroth order in $\alpha^{\prime}$ using the Scherk-Schwarz formalism [54]. We add hats to all the 10-dimensional objects (fields, indices, coordinates) and split the 10-dimensional world indices as $(\hat{\mu})=(\mu, \underline{z})$ and the 10-dimensional indices as $(\hat{a})=(a, z)$.

The Zehnbein and inverse-Zehnbein components $\hat{e}_{\hat{\mu}}{ }^{\hat{a}}$ and $\hat{e}_{\hat{a}}^{\hat{\mu}}$ can be put in an uppertriangular form by a local Lorentz transformation and, then, they can be decomposed in terms of the 9-dimensional Vielbein and inverse Vielbein components $e_{\mu}{ }^{a}, e_{a}{ }^{\mu}$, Kaluza-Klein (KK) vector $A_{\mu}$ and KK scalar $k$ as

$$
\left(\hat{e}_{\hat{\mu}}^{\hat{a}}\right)=\left(\begin{array}{cc}
e_{\mu}^{a} & k A_{\mu} \\
0 & k
\end{array}\right), \quad\left(\hat{e}_{\hat{a}}^{\hat{\mu}}\right)=\left(\begin{array}{cc}
e_{a}^{\mu} & -A_{a} \\
0 & k^{-1}
\end{array}\right),
$$


where $A_{a}=e_{a}{ }^{\mu} A_{\mu}$. We will always assume that all the 9-dimensional fields with Lorentz indices are 9-dimensional world tensors contracted with the 9-dimensional Vielbeins. For instance, the KK fields strength $F_{a b}$ is

$$
F_{a b}=e_{a}^{\mu} e_{b}{ }^{\nu} F_{\mu \nu}, \quad F_{\mu \nu} \equiv 2 \partial_{[\mu} A_{\nu]} .
$$

The components of the 10-dimensional spin connection $\hat{\omega}_{\hat{a} \hat{b} \hat{c}}$ decompose into those of the 9-dimensional one $\omega_{a b c}$ and $F_{a b}$ as

$$
\begin{array}{ll}
\hat{\omega}_{a b c}=\omega_{a b c}, & \hat{\omega}_{a b z}=\frac{1}{2} k F_{a b}, \\
\hat{\omega}_{z b c}=-\frac{1}{2} k F_{b c}, & \hat{\omega}_{z b z}=-\partial_{b} \ln k .
\end{array}
$$

Then, using the Palatini identity, it is not difficult to see that the first two terms in the action eq. (2.12) take the following 9-dimensional form (up to a total derivative):

$$
\begin{aligned}
& \int d^{10} \hat{x} \sqrt{|\hat{g}|} e^{-2 \hat{\phi}}\left\{\hat{R}-4(\partial \hat{\phi})^{2}\right\}= \\
& \quad \int d z \int d^{9} x \sqrt{|g|} e^{-2 \phi}\left\{R-4(\partial \phi)^{2}+(\partial \log k)^{2}-\frac{1}{4} k^{2} F^{2}\right\},
\end{aligned}
$$

where the 9-dimensional dilaton field is related to the 10-dimensional one by

$$
\phi \equiv \hat{\phi}-\frac{1}{2} \log k \text {. }
$$

At zeroth order in $\alpha^{\prime}$, the last term that we have to reduce is the kinetic term of the Kalb-Ramond 2-form $\sim \hat{H}^{(0) 2}$. Following Scherk and Schwarz, we consider the Lorentz components of the 3 -form field strength, because they are automatically gauge-invariant combinations. The $\hat{H}^{(0)}{ }_{a b z}$ components give

$$
\hat{H}^{(0)}{ }_{a b z}=k^{-1} e_{a}{ }^{\mu} e_{b}{ }^{\nu} \hat{H}^{(0)}{ }_{\mu \nu} \underline{z}=k^{-1} e_{a}{ }^{\mu} e_{b}{ }^{\nu} 2 \partial_{[\mu} \hat{B}_{\nu] \underline{z}} .
$$

It is, then, appropriate to define the zeroth-order "winding" ${ }^{14}$ vector field $B^{(0)}{ }_{\mu}$ and its field strength $G^{(0)}{ }_{\mu \nu}$ by

$$
B_{\mu}^{(0)} \equiv \hat{B}_{\mu \underline{z}}, \quad G_{\mu \nu}^{(0)} \equiv 2 \partial_{[\mu} B_{\nu]}^{(0)},
$$

so that

$$
\hat{H}^{(0)}{ }_{a b z}=k^{-1} G^{(0)}{ }_{a b} .
$$

The second gauge-invariant combination is

$$
\hat{H}^{(0)}{ }_{a b c}=e_{a}{ }^{\mu} e_{b}{ }^{\nu} e_{c}{ }^{\rho}\left(\hat{H}^{(0)}{ }_{\mu \nu \rho}-3 A_{[\mu} \hat{H}^{(0)}{ }_{\nu \rho]}\right),
$$

which suggests the definition

$$
H^{(0)}{ }_{\mu \nu \rho} \equiv \hat{H}^{(0)}{ }_{\mu \nu \rho}-3 A_{[\mu} \hat{H}_{\nu \rho] \underline{z}}^{(0)}=3 \partial_{[\mu} \hat{B}_{\nu \rho]}-6 A_{[\mu} \partial_{\nu} B_{\rho]}^{(0)} \cdot
$$

\footnotetext{
${ }^{14}$ This vector couples electrically to the string modes with non-vanishing winding numbers, just as the KK vector field couples to those with non-vanishing momentum in the internal direction.
} 
We could simply identify $\hat{B}_{\nu \rho}$ with the 9-dimensional Kalb-Ramond field, but it is customary (and convenient) to use a $\mathrm{T}$ duality-invariant definition. $\mathrm{T}$ duality will interchange KK momentum and winding, and therefore, will interchange $A_{\mu}$ with $B^{(0)}{ }_{\mu}$, modifying the Chern-Simons term in the above form of $H_{\mu \nu \rho}$. We can, however, rewrite it in the form

$$
H_{\mu \nu \rho}^{(0)}=3 \partial_{[\mu}\left(\hat{B}_{\nu \rho]}+A_{\mid \nu} B_{\rho]}^{(0)}\right)-\frac{3}{2} A_{[\mu} G_{\nu \rho]}^{(0)}-\frac{3}{2} B_{[\mu}^{(0)} F_{\nu \rho]},
$$

and identify the $\mathrm{T}$ duality-invariant 9-dimensional Kalb-Ramond 2-form

$$
B_{\mu \nu}^{(0)} \equiv \hat{B}_{\mu \nu}+A_{[\mu} B^{(0)}{ }_{\nu]},
$$

with the final result

$$
H_{\mu \nu \rho}^{(0)}=3 \partial_{[\mu} \hat{B}_{\nu \rho]}^{(0)}-\frac{3}{2} A_{[\mu} G_{\nu \rho]}^{(0)}-\frac{3}{2} B^{(0)}{ }_{[\mu} F_{\nu \rho]} .
$$

Then, after integrating over the length of the compact coordinate $z\left(2 \pi \ell_{s}\right.$ by convention) the 9-dimensional action to zeroth order in $\alpha^{\prime}$ takes the form

$$
S=\frac{g_{s}^{2}\left(2 \pi \ell_{s}\right)}{16 \pi G_{N}^{(10)}} \int d^{9} x \sqrt{|g|} e^{-2 \phi}\left\{R-4(\partial \phi)^{2}+(\partial \log k)^{2}-\frac{1}{4} k^{2} F^{2}-\frac{1}{4} k^{-2} G^{(0) 2}+\frac{1}{12} H^{(0) 2}\right\} .
$$

This action is invariant under the $\mathrm{T}$ duality transformations

$$
A_{\mu}^{\prime}=B_{\mu}^{(0)}, \quad B_{\mu}^{(0) \prime}=A_{\mu}, \quad k^{\prime}=1 / k .
$$

Taking into account the relations between the 10- and 9-dimensional fields, collected in appendix $\mathrm{A}$, it is easy to see that the above $\mathrm{T}$ duality transformations correspond to the following transformations of the 10-dimensional fields known as Buscher rules [37, 38]:

$$
\begin{array}{ll}
\hat{g}_{\underline{z z}}^{\prime}=1 / \hat{g}_{\underline{z z}}, & \hat{B}_{\mu \underline{z}}^{\prime}=\hat{g}_{\mu \underline{z}} / \hat{g}_{\underline{z z}}, \\
\hat{g}_{\mu \underline{z}}^{\prime}=\hat{B}_{\mu \underline{z}} / \hat{g}_{\underline{z z}}, & \hat{B}_{\mu \nu}^{\prime}=\hat{B}_{\mu \nu}+2 \hat{g}_{[\mu \mid \underline{z}} \hat{B}_{\nu] \underline{z}} / \hat{g}_{\underline{z z}}, \\
\hat{g}_{\mu \nu}^{\prime}=\hat{g}_{\mu \nu}-\left(\hat{g}_{\mu \underline{z}} \hat{g}_{\nu \underline{z}}-\hat{B}_{\mu \underline{z}} \hat{B}_{\nu \underline{z}}\right) / \hat{g}_{\underline{z z}}, & \hat{\phi}^{\prime}=\hat{\phi}-\frac{1}{2} \ln \left|\hat{g}_{\underline{z} z}\right| .
\end{array}
$$

\section{Dimensional reduction on $\mathrm{S}^{1}$ at $\mathcal{O}\left(\alpha^{\prime}\right)$}

The reduction of the first two terms in the effective action is not modified by the inclusion of $\alpha^{\prime}$ corrections. The definitions of 9-dimensional metric, dilaton and KK vector and scalar in terms of the 10-dimensional fields are not modified by them either. We expect modifications in the definitions of the 9-dimensional Kalb-Ramond 2-form and of the winding vector, though, because of the presence of the Lorentz and Yang-Mills Chern-Simons 3-forms in $\hat{H}^{(1)}$.

It is convenient to start by studying the dimensional reduction of the Yang-Mills fields. The Lorentz-indices decomposition of the gauge field is

$$
\begin{aligned}
& \hat{A}^{A}{ }_{z}=k^{-1} \hat{A}^{A}{ }_{\underline{z}}, \\
& \hat{A}^{A}{ }_{a}=e_{a}{ }^{\mu}\left(\hat{A}_{\mu}^{A}{ }_{\mu}-\hat{A}_{\underline{z}}^{A} A_{\mu}\right),
\end{aligned}
$$


which leads to the definition of the 9-dimensional adjoint scalars $\phi^{A}$ and gauge vectors

$$
\begin{aligned}
\varphi^{A} & \equiv k^{-1} \hat{A}_{\underline{z}}^{A}, \\
A^{A}{ }_{\mu} & \equiv \hat{A}^{A}{ }_{\mu}-\hat{A}_{\underline{z}}^{A} A_{\mu} .
\end{aligned}
$$

In terms of these variables, it is not difficult to see that the components of 10 dimensional gauge field strength are given by

$$
\begin{aligned}
\hat{F}^{A}{ }_{a z} & =\mathfrak{D}_{a} \varphi^{A}-\varphi^{A} \partial_{a} \log k, \\
\hat{F}^{A}{ }_{a b} & =F^{A}{ }_{a b}+k \varphi^{A} F_{a b},
\end{aligned}
$$

where $F^{A}{ }_{\mu \nu}$ is the standard Yang-Mills gauge field strength for the 9-dimensional gauge fields $A_{\mu}^{A}$.

The reduction of the first, second and fourth terms in the action eq. (2.12) gives (up to a total derivative)

$$
\begin{gathered}
\int d z \int d^{9} x \sqrt{|g|} e^{-2 \phi}\left\{R-4(\partial \phi)^{2}+\left(1+\frac{\alpha^{\prime}}{4} \varphi^{2}\right)(\partial \log k)^{2}+\frac{\alpha^{\prime}}{4}(\mathfrak{D} \varphi)^{2}\right. \\
\left.-\frac{1}{4}\left(1+\frac{\alpha^{\prime}}{2} \varphi^{2}\right) k^{2} F^{2}-\frac{\alpha^{\prime}}{8}\left(F_{A} \cdot F^{A}+2 \varphi_{A} F^{A} \cdot k F\right)\right\},
\end{gathered}
$$

where $\varphi^{2} \equiv \varphi_{A} \varphi^{A}, \mathfrak{D}_{\mu} \varphi^{A}=\partial_{\mu} \varphi^{A}+f_{B C}{ }^{A} A^{B}{ }_{\mu} \varphi^{C}$ etc.

Let us now consider the reduction of the Kalb-Ramond 3-form field strength $\hat{H}^{(1)}$, starting with the gauge-invariant combination

$$
\hat{H}_{a b z}^{(1)}=k^{-1} e_{a}{ }^{\mu} e_{b}{ }^{\nu} \hat{H}^{(1)}{ }_{\mu \nu \underline{z}}=k^{-1} e_{a}{ }^{\mu} e_{b}{ }^{\nu}\left\{2 \partial_{[\mu} \hat{B}_{\nu] \underline{z}}+\frac{\alpha^{\prime}}{4}\left(\hat{\omega}_{\mu \nu \underline{z}}^{\mathrm{YM}}+\hat{\omega}_{(-) \mu \nu \underline{z}}^{\mathrm{L}(0)}\right)\right\} .
$$

Using the above results for the Yang-Mills fields we find that

$$
\hat{\omega}^{\mathrm{YM}}{ }_{\mu \nu \underline{z}}=k \varphi_{A}\left(2 F_{\mu \nu}^{A}+\varphi^{A} k F_{\mu \nu}\right)-2 \partial_{[\mu \mid}\left(k \varphi_{A} A_{\mid \nu]}^{A}\right) .
$$

The last term is a total derivative that can be absorbed into the definition of the 9dimensional vector field $B^{(1)}{ }_{\mu}$ and the remaining terms are manifestly gauge-invariant 2 -forms.

We can use this result in the reduction of the Lorentz Chern-Simons 3-form; after all, the only difference with the Yang-Mills Chern-Simons 3-form is the gauge group, which now is the 10-dimensional Lorentz group. This is, nevertheless, an important difference because this group is broken down to the 9-dimensional Lorentz group times U(1) and we will have to take this fact into account in a second step.

In order to profit from the previous result, we introduce the following notation

$$
\begin{aligned}
\hat{A}^{\hat{a} \hat{b}} \hat{\mu} & \equiv \hat{\Omega}_{(-) \hat{\mu}}^{(0)} \hat{a} \hat{b} \\
\hat{F}^{\hat{a} \hat{b}}{ }_{\hat{\mu} \hat{\nu}} & \equiv \hat{R}_{(-) \hat{\mu} \hat{\mu}}^{(0)} \hat{\hat{\omega}} \hat{b} .
\end{aligned}
$$

Then, a straightforward application of eq. (4.6) gives

$$
\hat{\omega}_{(-) \mu \nu \underline{z}}^{\mathrm{L}(0)}=k \varphi_{\hat{b}}^{\hat{a}_{\hat{b}}}\left(2 F_{\hat{a} \mu \nu}^{\hat{b}}+\varphi_{\hat{a}}^{\hat{b}} k F_{\mu \nu}\right)-2 \partial_{[\mu \mid}\left(k \varphi_{\hat{b}}^{\hat{a}_{\hat{a}}} A_{\hat{a} \mid \nu]}^{\hat{b}}\right),
$$


where

$$
\begin{aligned}
\varphi^{\hat{a} \hat{b}} & =k^{-1} \hat{\Omega}_{(-) \underline{z}}^{(0)} \hat{a} \hat{b} \\
A^{\hat{a} \hat{b}}{ }_{\mu} & =\hat{\Omega}_{(-) \mu}^{(0)} \hat{a} \hat{b}-A_{\mu} \hat{\Omega}_{(-) \underline{z}}^{(0)} \hat{a} \hat{b}
\end{aligned}
$$

and where $F^{\hat{a} \hat{b}}{ }_{\mu \nu}$ is the standard field strength of the gauge field $A^{\hat{a} \hat{a}}{ }_{\mu}$ defined above. Decomposing now the Lorentz indices, we obtain

$\hat{\omega}_{(-) \mu \nu \underline{z}}^{\mathrm{L}(0)}=k \varphi_{b}^{a}\left(2 F^{b}{ }_{a \mu \nu}+\varphi^{b}{ }_{a} k F_{\mu \nu}\right)+2 k \varphi^{a z}\left(2 F_{a}^{z}{ }_{\mu \nu}+\varphi_{a}{ }^{z} k F_{\mu \nu}\right)-2 \partial_{[\mu \mid}\left[k\left(\varphi^{\hat{a}_{\hat{b}}} A^{\hat{b}}{ }_{\hat{a} \mid \nu]}\right)\right]$.

The components of these fields are

$$
\begin{aligned}
\varphi^{a b} & =-\frac{1}{2}\left(k F^{a b}+k^{-1} G^{(0) a b}\right), \\
\varphi^{a z} & =\partial^{a} \log k, \\
A^{a b}{ }_{\mu} & =\omega_{\mu}^{a b}-\frac{1}{2} H_{\mu}^{(0)}{ }^{a b} \equiv \Omega_{(-) \mu}^{(0)}{ }^{a b}, \\
A^{a z}{ }_{\mu} & =-\frac{1}{2}\left(k F_{\mu}^{a}-k^{-1} G_{\mu}^{(0)}{ }^{a}\right), \\
F^{a b}{ }_{\mu \nu} & =R_{(-) \mu \nu}^{(0)}{ }^{a b}-\frac{1}{2}\left(k F_{[\mu}^{a}-k^{-1} G_{[\mu}^{(0)}{ }^{a}\right)\left(k F_{\nu]}^{b}-k^{-1} G_{\nu]}^{(0)}{ }^{b}\right), \\
F^{a z}{ }_{\mu \nu} & =-\mathcal{D}_{(-)[\mu}^{(0)}\left(k F_{\nu]}^{a}-k^{-1} G_{\nu]}^{(0)}\right),
\end{aligned}
$$

where $R_{(-) \mu \nu}^{(0)}{ }^{a b}$ is the standard Lorentz curvature and $\mathcal{D}_{(-) \mu}^{(0)}$ is the standard Lorentzcovariant derivative with respect to the 9-dimensional torsionful spin connection $\Omega_{(-) \mu}^{(0)}{ }^{a b}$.

Replacing the above expressions in eq. (4.10) we obtain

$$
\begin{aligned}
\hat{\omega}_{(-) \mu \nu \underline{z}}^{\mathrm{L}(0)}= & -\frac{1}{2} k\left(k F_{b}^{a}{ }_{b}+k^{-1} G_{b}^{(0) a}\right)\left\{2 R_{(-) \mu \nu}^{(0)}{ }^{b}{ }_{a}-\left(k F_{[\mu \mid}^{b}-k^{-1} G_{[\mu}^{(0)}{ }^{b}\right)\left(k F_{\nu] a}-k^{-1} G_{\mid \nu] a}^{(0)}\right)\right. \\
& \left.-\frac{1}{2}\left(k F^{b}{ }_{a}+k^{-1} G_{a}^{(0) b}\right) k F_{\mu \nu}\right\}-2 \partial^{a} k\left[2 \mathcal{D}_{(-)[\mu}^{(0)}\left(k F_{\nu] a}-k^{-1} G_{\mid \nu] a}^{(0)}\right)-\partial_{a} k F_{\mu \nu}\right] \\
& -2 \partial_{[\mu \mid}\left[k\left(\varphi^{\hat{a}}{ }_{b} A_{\hat{a} \mid \nu]}^{\hat{b}}\right)\right],
\end{aligned}
$$

and

$$
\begin{aligned}
\hat{H}_{c d z}^{(1)}= & k^{-1} e_{c}{ }^{\mu} e_{d}{ }^{\nu}\left\{2 \partial_{[\mu}\left[\hat{B}_{\nu] \underline{z}}-\frac{\alpha^{\prime}}{4} k\left(\varphi_{A} A^{A}{ }_{\mid \nu]}+\varphi^{\hat{a}}{ }_{\hat{b}} A_{\hat{a} \mid \nu]}^{\hat{b}}\right)\right]\right. \\
& +\frac{\alpha^{\prime}}{4} k \varphi_{A}\left(2 F^{A}{ }_{\mu \nu}+\varphi^{A} k F_{\mu \nu}\right) \\
& -\frac{1}{2} k\left(k F^{a}{ }_{b}+k^{-1} G^{(0) a}{ }_{b}\right)\left[2 R_{(-) \mu \nu}^{(0)}{ }^{b}{ }_{a}-\left(k F_{[\mu \mid}{ }^{b}-k^{-1} G^{(0)}{ }_{[\mu}{ }^{b}\right)\left(k F_{\nu] a}-k^{-1} G^{(0)}{ }_{\mid \nu] a}\right)\right. \\
& \left.\left.-\frac{1}{2}\left(k F^{b}{ }_{a}+k^{-1} G^{(0) b}{ }_{a}\right) k F_{\mu \nu}\right]-2 \partial^{a} k\left[2 \mathcal{D}_{(-)[\mu}^{(0)}\left(k F_{\nu] a}-k^{-1} G^{(0)}{ }_{\mid \nu] a}\right)-\partial_{a} k F_{\mu \nu}\right]\right\} .
\end{aligned}
$$


Since the right-hand side has to be a gauge-invariant combination, it is natural to define the first-order in $\alpha^{\prime}$ winding vector and its field strength by

$$
\begin{aligned}
B_{\mu}^{(1)} & \equiv \hat{B}_{\mu \underline{z}}-\frac{\alpha^{\prime}}{4} k\left(\varphi_{A} A_{\mu}^{A}+\varphi_{\hat{b}^{\hat{a}}}^{\hat{A}_{\hat{a} \mu}^{\hat{b}}}\right) \\
& =\hat{B}_{\mu \underline{z}}-\frac{\alpha^{\prime}}{4}\left[\hat{A}_{\mu}^{A} \hat{A}_{A \underline{z}}+\hat{\Omega}_{(-) \mu}^{(0)} \hat{a}_{\hat{b}} \hat{\Omega}_{(-) \underline{z}}^{(0)} \hat{b}{ }_{\hat{a}}-A_{\mu}\left(\hat{A}_{\underline{z}}^{A} \hat{A}_{A \underline{z}}+\hat{\Omega}_{(-) \underline{a}}^{(0)} \hat{a}_{\hat{b}_{(-) \underline{z}}}^{(0)} \hat{b}^{\hat{a}}\right)\right], \\
G^{(1)}{ }_{\mu \nu} & \equiv 2 \partial_{[\mu} B_{\nu]}^{(1)} .
\end{aligned}
$$

Furthermore, it is also natural to define the combinations

$$
K_{\mu \nu}^{( \pm)} \equiv k F_{\mu \nu} \pm k^{-1} G^{(0)}{ }_{\mu \nu} .
$$

$K^{(+)}{ }_{\mu \nu}$ is invariant under the zeroth-order $\mathrm{T}$ duality transformations eq. (3.15) while $K^{(-)}{ }_{\mu \nu}$ gets a minus sign under the same transformations. With this notation, we can finally write

$$
\begin{aligned}
\hat{H}_{a b z}^{(1)}= & k^{-1} G_{a b}^{(1)}+\frac{\alpha^{\prime}}{4}\left\{2 \varphi_{A} F_{a b}^{A}+\left[\varphi^{2}-\frac{1}{4} K^{(+) 2}+2(\partial \log k)^{2}\right] k F_{a b}\right. \\
& \left.\left.+R_{(-) a b}^{(0)}{ }^{c d} K^{(+)}{ }_{c d}-\frac{1}{2} K^{(-)}{ }_{a}^{c} K^{(-)} b_{b}{ }^{(} K^{(+)}{ }_{c d}-4 \mathcal{D}_{(-)[a}^{(0)} K^{(-)} b\right] c \partial^{c} \log k\right\} .
\end{aligned}
$$

This term contributes as $-\frac{1}{4} \hat{H}^{(1)}{ }_{a b z} \hat{H}^{(1) a b}$, which, at first order in $\alpha^{\prime}$ gives

$$
\begin{aligned}
-\frac{1}{4} \hat{H}_{a b z}^{(1)} \hat{H}_{z}^{(1) a b}= & -\frac{1}{4} k^{-2} G^{(1) 2} \\
& -\frac{\alpha^{\prime}}{8}\left\{2 \varphi_{A} F^{A} \cdot k^{-1} G^{(0)}+\left[\varphi^{2}-\frac{1}{4} K^{(+) 2}+(\partial \log k)^{2}\right] F \cdot G^{(0)}\right. \\
& +k^{-1} R_{(-) a b}^{(0)}{ }^{c d} K^{(+)}{ }_{c d} G^{(0) a b}-\frac{1}{2} k^{-1} G^{(0) a b} K^{(-)}{ }_{a}^{c} K^{(-)} b^{d} K^{(+)} c d \\
& \left.-4 k^{-1} G^{(0) a b} \mathcal{D}_{(-)[a}^{(0)} K^{(-)}{ }_{b] c} \partial^{c} \log k\right\} .
\end{aligned}
$$

Let us now move to the gauge-invariant combination $\hat{H}^{(1)}{ }_{a b c}$, that we will identify with the 9-dimensional Kalb-Ramond 3-form field strength. Using the zeroth-order result, we get

$$
\hat{H}^{(1)}{ }_{a b c}=H^{(0)}{ }_{a b c}+\frac{\alpha^{\prime}}{4}\left(\hat{\omega}^{\mathrm{YM}}{ }_{a b c}+\hat{\omega}^{\mathrm{L}(0)}{ }_{a b c}\right) .
$$

Using eqs. (4.3) it is almost immediately seen that

$$
\hat{\omega}^{\mathrm{YM}}{ }_{a b c}=\omega^{\mathrm{YM}}{ }_{a b c}+3 k \varphi_{A} F_{[a b} A_{c]}^{A} .
$$

The last term has to be integrated by parts, and the final result is

$$
\hat{\omega}^{\mathrm{YM}}{ }_{a b c}=\omega^{\mathrm{YM}}{ }_{a b c}+6 e_{a}^{\mu} e_{b}^{\nu} e_{c}^{\rho}\left[\partial_{[\mu}\left(k A_{\nu \mid} \varphi_{A} A_{\mid \rho]}^{A}\right)+A_{[\mu} \partial_{\nu \mid}\left(k \varphi_{A} A_{\mid \rho]}^{A}\right)\right] .
$$


The second term in the above expression, a total derivative, will combine with $\hat{B}_{\mu \nu}$ (and terms coming from $\hat{\omega}^{\mathrm{L}(0)}{ }_{a b c}$ ) to give $B^{(1)}{ }_{\mu \nu}$ and the third term, as we know, combines with $\hat{B}_{\mu \underline{z}}$ (and terms coming from $\hat{\omega}^{\mathrm{L}(0)}{ }_{a b c}$ ) to give $B^{(1)}{ }_{\mu}$.

The above result can be applied to $\hat{\omega}^{\mathrm{L}(0)}{ }_{a b c}$, using the definitions eq. (4.9). We get

$$
\begin{aligned}
\hat{\omega}^{\mathrm{L}(0)}{ }_{a b c}= & \omega^{\mathrm{L}(0)}{ }_{a b c}+e_{a}^{\mu} e_{b}{ }^{\nu} e_{c}{ }^{\rho}\left[\partial_{[\mu} K_{\nu}^{(-)}{ }_{\nu}^{e} K_{\rho] e}^{(-)}+6 \partial_{[\mu}\left(k A_{\nu \mid} \varphi_{\hat{f}}^{\hat{e}} A_{\hat{e} \mid \rho]}^{\hat{f}}\right)\right. \\
& \left.+6 A_{[\mu} \partial_{\nu \mid}\left(k \varphi^{\hat{e}}{ }_{\hat{f}} A^{\hat{f}}{ }_{\hat{e} \mid \rho]}\right)\right] .
\end{aligned}
$$

Defining

$$
B_{\mu \nu}^{(1)} \equiv \hat{B}_{\mu \nu}+A_{[\mu}\left[\hat{B}_{\nu] \underline{z}}+\frac{\alpha^{\prime}}{4} k\left(\hat{A}_{\mid \nu]}^{A} \hat{A}_{A \underline{z}}+\hat{\Omega}_{(-) \mid \nu]}^{(0)} \hat{a}_{\hat{b}} \hat{\Omega}_{(-) \underline{z}}^{(0)} \hat{b}\right)\right]
$$

we find

$$
\begin{aligned}
\hat{H}^{(1)}{ }_{a b c}= & H^{(1)}{ }_{a b c}, \\
H_{\mu \nu \rho}^{(1)} \equiv & 3 \partial_{[\mu} B_{\nu \rho]}^{(1)}-\frac{3}{2} A_{[\mu} G_{\nu \rho]}^{(1)}-\frac{3}{2} B_{[\mu}^{(1)} F_{\nu \rho]} \\
& +\frac{\alpha^{\prime}}{4}\left(\omega_{\mu \nu \rho}^{\mathrm{YM}}+\omega_{(-) \mu \nu \rho}^{\mathrm{L}(0)}+\mathcal{D}_{(-)[\mu} K_{\nu}^{(-)}{ }_{\nu}^{e} K_{\rho] e}^{(-)}\right) .
\end{aligned}
$$

Summarizing, the reduction of all the terms in the action but the last one gives, to $\mathcal{O}\left(\alpha^{\prime}\right)$

$$
\begin{aligned}
\int d z & \int d^{9} x \sqrt{|g|} e^{-2 \phi}\left\{R-4(\partial \phi)^{2}+\left(1+\frac{\alpha^{\prime}}{4} \varphi^{2}\right)(\partial \log k)^{2}+\frac{\alpha^{\prime}}{4}(\mathfrak{D} \varphi)^{2}\right. \\
& -\frac{1}{4}\left(1+\frac{\alpha^{\prime}}{2} \varphi^{2}\right) k^{2} F^{2}+\frac{1}{12} H^{(1) 2}-\frac{1}{4} k^{-2} G^{(1) 2}-\frac{\alpha^{\prime}}{8}\left[F_{A} \cdot F^{A}+2 \varphi_{A} F^{A} \cdot K^{(+)}\right. \\
& +\left[\varphi^{2}-\frac{1}{4} K^{(+) 2}+2(\partial \log k)^{2}\right] F \cdot G^{(0)}+R_{(-) a b}^{(0)}{ }^{c d} K^{(+)}{ }_{c d} k^{-1} G^{(0) a b} \\
& \left.\left.-\frac{1}{2} k^{-1} G^{(0) a b} K^{(-)}{ }_{a}^{c} K^{(-)}{ }_{b}^{d} K^{(+)}{ }_{c d}-4 k^{-1} G^{(0) a b} \mathcal{D}_{(-)[a}^{(0)} K_{b] c}^{(-)} \partial^{c} \log k\right]\right\}
\end{aligned}
$$

Now, we must deal with the last term. We deal with it in the same way as we dealt with the Yang-Mills kinetic term:

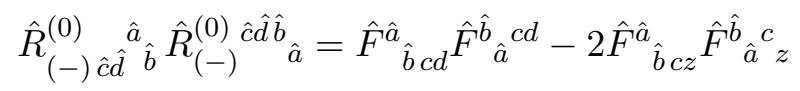

$$
\begin{aligned}
& =\left(F_{\hat{b} c d}^{\hat{a}}+k \varphi_{\hat{b}}^{\hat{a}} F_{c d}\right)\left(F_{\hat{a}}^{\hat{b}_{\hat{a}}}{ }^{\hat{b}^{\hat{b}}}+k \varphi_{\hat{a}}^{\hat{b}} F^{c d}\right) \\
& -2\left(\mathcal{D}_{c} \varphi_{\hat{b}}^{\hat{a}}-\varphi_{\hat{b}}^{\hat{a}} \partial_{c} \log k\right)\left(\mathcal{D}^{c} \varphi_{\hat{a}}^{\hat{b}}-\varphi_{\hat{a}}^{\hat{b}} \partial^{c} \log k\right) \text {. }
\end{aligned}
$$

The Lorentz-covariant derivatives in the last line must be taken with respect to the connection $A^{\hat{a} \hat{b}}{ }_{\mu}$, which means that the $a b$ components contain contributions from $A^{a z}{ }_{\mu}$ etc. Taking this fact into account, if we split the hatted indices into unhatted indices and $z$ 
components, we get

$$
\begin{aligned}
& \left(F_{b \mu \nu}^{a}+k \varphi_{b}^{a} F_{\mu \nu}\right)\left(F^{b}{ }_{a}{ }^{\mu \nu}+k \varphi^{b}{ }_{a} F^{\mu \nu}\right)+2\left(F^{a z}{ }_{\mu \nu}+k \varphi^{a z} F_{\mu \nu}\right)\left(F_{a}{ }^{z \mu \nu}+k \varphi_{a}{ }^{z} F^{\mu \nu}\right) \\
& -2\left(\mathcal{D}_{c} \varphi^{a}{ }_{b}-A^{a z}{ }_{c} \varphi_{b}{ }^{z}+A_{b}{ }^{z}{ }_{c} \varphi^{a z}-\varphi^{a}{ }_{b} \partial_{c} \log k\right)\left(\mathcal{D}^{c} \varphi^{b}{ }_{a}-A^{b z}{ }_{c} \varphi_{a}{ }^{z}+A_{a}{ }_{c}{ }_{c} \varphi^{b z}-\varphi^{b}{ }_{a} \partial^{c} \log k\right) \\
& -4\left(\mathcal{D}_{c} \varphi^{a z}+A^{b z}{ }_{c} \varphi^{a}{ }_{b}-\varphi^{a z} \partial_{c} \log k\right)\left(\mathcal{D}^{c} \varphi_{a}{ }^{z}+A^{b z} \varphi_{a b}-\varphi_{a}{ }^{z} \partial^{c} \log k\right),
\end{aligned}
$$

where, now $\mathcal{D}_{c}$ is the Lorentz-covariant derivative with respect to the connection $A^{a b}{ }_{\mu}$.

Substituting the components $A^{a b}{ }_{\mu}, A^{a z}{ }_{\mu}, \varphi^{a b}, \varphi^{a z}$ by their values, we get

$$
\begin{aligned}
& \left(R_{(-) \mu \nu}^{(0)}{ }_{b}^{a}-\frac{1}{2} K_{[\mu}^{(-)}{ }^{a} K^{(-)}{ }_{\nu] b}-\frac{1}{2} K^{(+)}{ }_{b} k F_{\mu \nu}\right)\left(R_{(-)}^{(0) \mu \nu b}{ }_{a}-\frac{1}{2} K^{(-) \mu b} K^{(-) \nu}{ }_{a}-\frac{1}{2} K^{(+) b}{ }_{a} k F^{\mu \nu}\right) \\
& +2\left(\mathcal{D}_{(-)[\mu}^{(0)} K_{\nu] a}^{(-)}{ }^{-} \partial_{a} \log k k F_{\mu \nu}\right)\left(\mathcal{D}_{(-)}^{(0)[\mu \mid} K^{(-) \mid \nu] a}-\partial^{a} \log k k F^{\mu \nu}\right) \\
& +\frac{1}{2}\left(\mathcal{D}_{(-)}^{(0)} K^{(+) a b}-2 K^{(-) c[a} \partial^{b]} \log k-K^{(+) a b} \partial^{c} \log k\right) \\
& \left(\mathcal{D}_{(-) c}^{(0)} K^{(+)}{ }_{a b}-2 K^{(-)}{ }_{c[a} \partial_{b]} \log k-K^{(+)}{ }_{a b} \partial_{c} \log k\right) \\
& -4\left(\mathcal{D}_{(-)}^{(0)}{ }^{c} \partial^{a} \log k-\frac{1}{4} K^{(-) c b} K^{(+)}{ }_{b}^{a}-\partial^{a} \log k \partial^{c} \log k\right) \\
& \left(\mathcal{D}_{(-) c}^{(0)} \partial_{a} \log k-\frac{1}{4} K^{(-)}{ }_{c}^{b} K^{(+)}{ }_{b a}-\partial_{a} \log k \partial_{c} \log k\right) .
\end{aligned}
$$

Operating, we finally get

$$
\begin{aligned}
& \hat{R}_{(-) \hat{c} \hat{d} \hat{b}}^{\hat{b}} \hat{R}_{(-)}^{(0) \hat{c} \hat{d} \hat{b}}{ }_{\hat{a}}=R_{(-) \mu \nu}^{(0)}{ }^{a} b R_{(-)}^{(0) \mu \nu b}{ }_{a}+R_{(-) \mu \nu}^{(0)}{ }^{a b} K^{(-) \mu}{ }_{a} K^{(-) \nu}{ }_{b}+R_{(-) \mu \nu}^{(0)}{ }^{a b} K^{(+)}{ }_{a b} k F^{\mu \nu} \\
& +\frac{1}{4} K^{(-)}{ }_{[\mu \mid}^{a} K^{(-)}{ }_{a}^{\nu} K^{(-)}{ }_{\mid \nu]}^{b} K^{(-)} b^{\mu}-\frac{1}{2} K^{(-)}{ }_{\mu a} K^{(-)}{ }_{\nu b} K^{(+) a b} k F^{\mu \nu} \\
& -\frac{1}{4}\left(K^{(+)}\right)^{2} k^{2} F^{2}+2 \mathcal{D}_{(-)}^{(0)}\left[\mu\left|K^{(-) \mid \nu] a} \mathcal{D}_{(-)[\mu \mid}^{(0)} K^{(-)}\right| \nu\right] a \\
& -4 \mathcal{D}_{(-)}^{(0)} \mu K^{(-) \nu a} \partial_{a} \log k k F_{\mu \nu}+2(\partial \log k)^{2} k^{2} F^{2} \\
& +\frac{1}{2} \mathcal{D}_{(-)}^{(0)}{ }^{c} K^{(+) a b} \mathcal{D}_{(-) c}^{(0)} K^{(+)}{ }_{a b}-2 \mathcal{D}_{(-)}^{(0)}{ }^{c} K^{(+) a b} K^{(-)}{ }_{c a} \partial_{b} \log k \\
& -\mathcal{D}_{(-)}^{(0)}{ }^{c} K^{(+) a b} K^{(+)}{ }_{a b} \partial_{c} \log k+2 K^{(-) c[a} \partial^{b]} \log k K^{(-)}{ }_{c a} \partial_{b} \log k \\
& +2 K^{(-) c a} \partial^{b} \log k K^{(+)}{ }_{a b} \partial_{c} \log k+\frac{1}{2}\left(K^{(+)}\right)^{2}(\partial \log k)^{2} \\
& -4 \mathcal{D}_{(-)}^{(0)}{ }^{c} \partial^{a} \log k \mathcal{D}_{(-) c}^{(0)} \partial_{a} \log k+2 \mathcal{D}_{(-)}^{(0)} c \partial^{a} \log k K^{(-)}{ }_{c}^{b} K^{(+)}{ }_{b a} \\
& -2 K^{(-) c b} K^{(+)}{ }_{b}^{a} \partial_{c} \log k \partial_{a} \log k-\frac{1}{4} K^{(-) a}{ }_{b} K^{(+) b}{ }_{c} K^{(-)}{ }_{d} K^{(+) d}{ }_{a} \\
& +8 \mathcal{D}_{(-)}^{(0)}{ }^{c} \partial^{a} \log k \partial_{a} \log k \partial_{c} \log k-4\left((\partial \log k)^{2}\right)^{2} .
\end{aligned}
$$


With all these terms, the action takes the form

$$
\begin{aligned}
S= & \frac{g_{s}^{2}\left(2 \pi \ell_{s}\right)}{16 \pi G_{N}^{(10)}} \int d^{9} x \sqrt{|g|} e^{-2 \phi}\left\{R-4(\partial \phi)^{2}+\frac{\alpha^{\prime}}{4}(\mathfrak{D} \varphi)^{2}-\partial_{a} k^{-1} \partial^{a} k^{(1)}\right. \\
& -\frac{1}{4} k^{(1) 2} F^{2}-\frac{1}{4} k^{-2} G^{(1) 2}+\frac{1}{2}\left(1-k^{(1)} k^{-1}\right) F \cdot G^{(1)}+\frac{1}{12} H^{(1) 2} \\
& -\frac{\alpha^{\prime}}{8}\left[F_{A} \cdot F^{A}+R_{(-)}^{(0) a} b \cdot R_{(-)}^{(0) b}{ }_{a}+R_{(-) a b}^{(0)}{ }_{c} d\left(K^{(-) a}{ }_{c} K^{(-) b}{ }_{d}+K^{(+) a b} K^{(+)}{ }_{c d}\right)\right. \\
& +2 \varphi_{A} F^{A} \cdot K^{(+)} \\
& -\frac{3}{4} K^{(+) a}{ }_{b} K^{(-) b}{ }_{c} K^{(+) c}{ }_{d} K^{(-) d}{ }_{a}+\frac{1}{8} K^{(-) a}{ }_{b} K^{(-) b}{ }_{c} K^{(-) c}{ }_{d} K^{(-)}{ }_{a}-\frac{1}{8}\left(K^{(-)} \cdot K^{(-)}\right)^{2} \\
& -4 K^{(+) a b} \mathcal{D}_{(-) a}^{(0)} K^{(-)}{ }_{b c} \partial^{c} \log k-2 K^{(-) a b} \mathcal{D}_{(-) a}^{(0)} K^{(+)}{ }_{b c} \partial^{c} \log k \\
& +2 \mathcal{D}_{(-)}^{(0)[a \mid} K^{(-) \mid b] c} \mathcal{D}_{(-)[a \mid}^{(0)} K^{(-)}{ }_{\mid b] c}+\frac{1}{2} \mathcal{D}_{(-)}^{(0)}{ }^{c} K^{(+) a b} \mathcal{D}_{(-) c}^{(0)} K^{(+)}{ }_{a b} \\
& -4 \mathcal{D}_{(-)}^{(0) c} \partial^{a} \log k \mathcal{D}_{(-) c}^{(0)} \partial_{a} \log k+2 K^{(-) a c} K^{(+)}{ }_{c}{ }^{b} \mathcal{D}_{(-) a}^{(0)} \partial_{b} \log k \\
& \left.\left.+2 K^{(-) c[a} \partial^{b]} \log k K^{(-)}{ }_{c a} \partial_{b} \log k\right]\right\},
\end{aligned}
$$

where we have defined

$$
k^{(1)} \equiv k\left[1+\frac{\alpha^{\prime}}{4}\left(\varphi^{2}-\frac{1}{4} K^{(+)} 2+2(\partial \log k)^{2}\right)\right]
$$

and we have added some $\mathcal{O}\left(\alpha^{\prime 2}\right)$ terms in order to obtain nicer or simpler expressions.

\subsection{T duality}

All the $\mathcal{O}\left(\alpha^{\prime}\right)$ terms of the reduced action eq. (4.29) are invariant under the zeroth-order $\mathrm{T}$ duality transformations eqs. (3.15), and the whole action is invariant to $\mathcal{O}\left(\alpha^{\prime}\right)$ under the transformations

$$
A_{\mu}^{\prime}=B_{\mu}^{(1)}, \quad B_{\mu}^{(1) \prime}=A_{\mu}, \quad k^{\prime}=1 / k^{(1)},
$$

which reduce to the zeroth-order ones in eqs. (3.15) when we set $\alpha^{\prime}=0$. Furthermore, observe that

$$
\begin{aligned}
k^{(1) \prime} & =k^{\prime}\left[1+\frac{\alpha^{\prime}}{4}\left(\varphi^{2}-\frac{1}{4} K^{(+) 2}+2(\partial \log k)^{2}\right)\right] \\
& =k^{(1)-1}\left[1+\frac{\alpha^{\prime}}{4}\left(\varphi^{2}-\frac{1}{4} K^{(+) 2}+2(\partial \log k)^{2}\right)\right] \\
& =k^{-1}\left[1+\mathcal{O}\left(\alpha^{\prime 2}\right)\right] .
\end{aligned}
$$


Using the relation between the higher- and lower-dimensional fields, these transformations can be expressed in terms of the higher-dimensional ones in the form

$$
\begin{aligned}
& \hat{g}_{\mu \nu}^{\prime}=\hat{g}_{\mu \nu}+\frac{\hat{g}_{\underline{z} \underline{\mathfrak{G}^{(1)}}} \hat{\underline{z}}_{\mu}^{\hat{\mathfrak{G}}^{(1)} \underline{z} \nu}}{\hat{\mathfrak{G}}^{(1)} \underline{\underline{z} z}_{\underline{z}}^{2}}-\frac{2 \hat{\mathfrak{G}}^{(1)} \underline{z}_{\mu} \hat{g}_{\nu) \underline{z}}}{\hat{\mathfrak{G}}^{(1)} \underline{z}_{z}}, \\
& \hat{B}_{\mu \nu}^{\prime}=\hat{B}_{\mu \nu}-\frac{\hat{\mathfrak{G}}^{(1)}{ }_{\underline{z}}\left[\mu \hat{\mathfrak{G}}^{(1)} \nu\right] \underline{z}}{\hat{\mathfrak{G}}^{(1)} \underline{z z}}, \\
& \hat{g}_{\underline{z} \mu}^{\prime}=-\frac{\hat{g}_{\underline{z} \mu}}{\hat{\mathfrak{G}}^{(1)} \underline{z z}}+\frac{\hat{g}_{\underline{z z}} \hat{\mathfrak{G}}^{(1)} \underline{z \mu}}{\hat{\mathfrak{G}}^{(1)} \underline{\underline{z} z}}, \quad \quad \hat{B}_{\underline{z} \mu}^{\prime}=-\frac{\hat{B}_{\underline{z} \mu}}{\hat{\mathfrak{G}}^{(1)} \underline{z z}}-\frac{\hat{\mathfrak{G}}^{(1)} \underline{z} \mu}{\hat{\mathfrak{G}}^{(1)} \underline{z z}}, \\
& \hat{g}_{\underline{z z}}^{\prime}=\frac{\hat{g}_{\underline{z z}}}{\left.\hat{\mathfrak{G}}^{(1) 2}\right)_{\underline{z}}}, \quad \quad e^{-2 \hat{\phi}^{\prime}}=e^{-2 \hat{\phi}}\left|\hat{\mathfrak{G}}^{(1)} \underline{z z}\right|, \\
& \hat{A}_{\underline{z}}^{\prime A}=-\frac{\hat{A}_{\underline{z}}^{A}}{\hat{\mathfrak{G}}^{(1)} \underline{z z}}, \quad \quad \hat{A}^{\prime A}{ }_{\mu}=\hat{A}_{\mu}^{A}-\frac{\hat{A}^{A}{ }_{\underline{z}} \hat{\mathfrak{G}}^{(1)}{ }_{\underline{z}} \mu}{\hat{\mathfrak{G}}^{(1)} \underline{z z}}
\end{aligned}
$$

where the tensor $\hat{\mathfrak{G}}^{(1)} \hat{\mu} \hat{\nu}$ is defined by

$$
\hat{\mathfrak{G}}^{(1)} \hat{\mu} \hat{\nu} \equiv \hat{g}_{\hat{\mu} \hat{\nu}}-\hat{B}_{\hat{\mu} \hat{\nu}}-\frac{\alpha^{\prime}}{4}\left\{\hat{A}_{\hat{\mu}}^{A A} \hat{A}_{A \hat{\nu}}+\hat{\Omega}_{(-) \hat{\mu}}^{(0)} \hat{b} \hat{\Omega}_{(-) \hat{\nu}}^{(0)} \hat{b}\right\}
$$

These are the $\alpha^{\prime}$-corrected Buscher rules first found in ref. [42] and later rediscovered elsewhere [43, 44].

It is well known that $\mathcal{N}=1, d=10$ supergravity $[55,56]$ coupled to $n_{V}$ Abelian vector multiplets $[55,56]$ and dimensionally reduced on a $\mathrm{T}^{n}$ has a global $\mathrm{O}\left(n, n+n_{V}\right)$ symmetry which was shown in ref. [10] to be related to string T duality. In the case at hand, the YM vectors are, generically, non-Abelian, which reduces the symmetry to just $\mathrm{O}(n, n)$ [47] or just $\mathrm{O}(1,1)$ here. This group consists of the discrete transformation that give rise to the Buscher rules eq. (4.31) and rescalings of just certain lower-dimensional fields:

$$
A_{\mu}^{\prime}=\lambda^{-1} A_{\mu}, \quad B_{\mu}^{(1) \prime}=\lambda B_{\mu}^{(1) \prime}, \quad k^{\prime}=\lambda k .
$$

Under these rescalings $K^{ \pm}, H^{(1)}$ and the Lorentz curvature terms remain invariant while

$$
k^{(1) \prime}=\lambda k^{(1)} .
$$

It can be checked that the dimensionally-reduced action eq. (4.29) is invariant under these transformations and, therefore, under the whole $\mathrm{O}(1,1)$ group.

We observe that the kinetic term of the KK and winding vectors is the sum of two separately $\mathrm{O}(1,1)$-invariant terms

$$
-\frac{1}{4}\left(F_{\mu \nu}, G_{\mu \nu}^{(1)}\right)\left(\begin{array}{cc}
k^{(1) 2} & 0 \\
0 & 1 / k^{2}
\end{array}\right)\left(\begin{array}{c}
F^{\mu \nu} \\
G^{(1) \mu \nu}
\end{array}\right)+\frac{1}{2}\left(1-k^{(1)} / k\right) F \cdot G^{(1)},
$$

and that the diagonal kinetic matrix transforms consistently under $\mathrm{O}(1,1)$ transformations even though, as different to the zeroth-order case, the kinetic matrix is not an $\mathrm{O}(1,1)$ matrix itself. The consistency is related to the fact that it is part of a $\mathrm{O}\left(1,1+n_{V}\right)$ matrix. 


\section{Entropy formula}

We can use the dimensionally reduced action we have obtained to calculate the entropy of some $d$-dimensional heterotic string black holes using the Iyer-Wald prescription [25, 26]. These black holes must be solutions of the theory defined by the action eq. (4.29) understood as a $d$-dimensional action. Therefore, they must be solutions of the theory defined by the action eq. (2.12) understood as a $(d+1)$-dimensional action ${ }^{15}$ admitting an isometry. Since this $(d+1)$-dimensional action can be obtained from the 10-dimensional one by a trivial compactification on a $10-(d+1)$-dimensional torus, the metrics of the 10-dimensional solutions corresponding to the $d$-dimensional black holes are the direct products of nontrivial $(d+1)$-dimensional metrics and the metric of a $10-(d+1)$-dimensional torus. The non-extremal 4-dimensional Reissner-Nordström black hole of ref. [21] or the heterotic version of the 5-dimensional Strominger-Vafa black hole of ref. [17] are two interesting examples of this kind of solution.

Applying directly the Iyer-Wald prescription to the $d$-dimensional action eq. (4.29) we obtain the following entropy formula expressed in string-frame variables:

$$
\begin{aligned}
S= & -2 \pi \int_{\Sigma} d^{d-2} x \sqrt{|h|} \frac{\partial \mathcal{L}}{\partial R_{a b c d}} \epsilon_{a b} \epsilon_{c d}, \\
\frac{\partial \mathcal{L}}{\partial R_{a b c d}}= & \frac{e^{-2\left(\phi-\phi_{\infty}\right)}}{16 \pi G_{N}^{(d)}}\left\{g^{a b, c d}-\frac{\alpha^{\prime}}{8}\left[H^{(0) a b g}\left(\omega_{g}^{c d}-H_{g}^{(0) c d}\right)\right.\right. \\
& \left.\left.-2 R_{(-)}^{(0) a b c d}+K^{(-)[a \mid c} K^{(-) \mid b] d}+K^{(+) a b} K^{(+) c d}\right]\right\},
\end{aligned}
$$

where $|h|$ is the absolute value of the determinant of the metric induced over the event horizon, $g^{a b, c d}=\frac{1}{2}\left(g^{a c} g^{b d}-g^{a d} g^{b c}\right), \epsilon^{a b}$ is the event horizon's binormal normalized so that $\epsilon_{a b} \epsilon^{a b}=-2$ and $R_{a b c d}$ is the Riemann tensor.

\subsection{The Wald entropy of the $\alpha^{\prime}$-corrected Strominger-Vafa black hole}

The entropy formula eq. (5.2b) has been shown in ref. [21] to give an entropy which is related to the Hawking temperature by the thermodynamic relation

$$
\frac{\partial S}{\partial M}=\frac{1}{T}
$$

\footnotetext{
${ }^{15}$ The constant in front of the action should now contain the volume of a $(10-d)$-dimensional torus instead of that of circle, that is

$$
\frac{g_{s}^{2}\left(2 \pi \ell_{s}\right)^{10-d}}{16 \pi G_{N}^{(10)}}=\frac{\left(g_{s}^{(d)}\right)^{2}}{16 \pi G_{N}^{(d)}},
$$

where $g_{s}^{(d)}$ is the $d$-dimensional string coupling constant or the vacuum expected value of the $d$-dimensional dilaton $\left\langle e^{\phi}\right\rangle=e^{\phi_{\infty}}$ and $G_{N}^{(d)}$ the $d$-dimensional Newton constant. The relations of the 10-dimensional and $d$-dimensional ones with the volume of the $(10-d)$-dimensional compact space, $V_{10-d}$ is

$$
\begin{aligned}
g_{s}^{2} & =V_{10-d} /\left(2 \pi \ell_{s}\right)^{10-d} g_{s}^{(d) 2}, \\
G_{N}^{(10)} & =G_{N}^{(d)} V_{10-d} .
\end{aligned}
$$


for the particular case of $\alpha^{\prime}$-corrected, 4-dimensional, non-extremal Reissner-Nordström black holes. In this section we want to recalculate the Wald entropy of the $\alpha^{\prime}$-corrected Strominger-Vafa black hole. Being an extremal black hole, we will not be able to check that the entropy obtained is related to the temperature as above, but, instead, we will be able to compare with other results obtained in the literature and with the microscopic calculations.

The 5-dimensional $\alpha^{\prime}$-corrected Strominger-Vafa black hole corresponds to the 10dimensional solution of the Heterotic Superstring effective action [17, 18]

$$
\begin{aligned}
d \hat{s}^{2} & =\frac{2}{\mathcal{Z}_{-}} d u\left(d v-\frac{1}{2} \mathcal{Z}_{+} d u\right)-\mathcal{Z}_{0}\left(d \rho^{2}+\rho^{2} d \Omega_{(3)}^{2}\right)-d y^{i} d y^{i}, \quad i=1, \ldots, 4, \\
\hat{H}^{(1)} & =d \mathcal{Z}_{-}^{-1} \wedge d u \wedge d v+\star_{4} d \mathcal{Z}_{0}, \\
e^{-2 \hat{\phi}} & =e^{-2 \hat{\phi}_{\infty}} \mathcal{Z}_{-} / \mathcal{Z}_{0}
\end{aligned}
$$

where $\star_{4}$ stands for the Hodge dual in the 4-dimensional Euclidean space with metric $d \rho^{2}+\rho^{2} d \Omega_{(3)}^{2}$, and where the $\mathcal{Z}$ functions take the values ${ }^{16}$

$$
\begin{aligned}
& \mathcal{Z}_{0}=1+\frac{\tilde{q}_{0}}{\rho^{2}}-\alpha^{\prime} \frac{\rho^{2}+2 \tilde{q}_{0}}{\left(\rho^{2}+\tilde{q}_{0}\right)^{2}}+\mathcal{O}\left(\alpha^{\prime 2}\right), \\
& \mathcal{Z}_{-}=1+\frac{\tilde{q}_{-}}{\rho^{2}}+\mathcal{O}\left(\alpha^{\prime 2}\right), \\
& \mathcal{Z}_{+}=1+\frac{\tilde{q}_{+}}{\rho^{2}}+2 \alpha^{\prime} \frac{\tilde{q}_{+}\left(\rho^{2}+\tilde{q}_{0}+\tilde{q}_{-}\right)}{\tilde{q}_{0}\left(\rho^{2}+\tilde{q}_{0}\right)\left(\rho^{2}+\tilde{q}_{-}\right)}+\mathcal{O}\left(\alpha^{\prime 2}\right) .
\end{aligned}
$$

Compactifying this solution in a $\mathrm{T}^{4}$ parameterized by the coordinates $y_{i}$ is trivial. Then, we just have to compactify the resulting 6 -dimensional solution to $d=5$ using the results obtained here along the coordinate $z \equiv u / k_{\infty}$, where $k_{\infty}$ is the asymptotic value of the KK scalar $k$. It is helpful to rewrite the 6-dimensional solution in the form

$$
\begin{aligned}
d \hat{s}^{2} & =\frac{1}{\mathcal{Z}_{+} \mathcal{Z}_{-}} d t^{2}-\mathcal{Z}_{0}\left(d \rho^{2}+\rho^{2} d \Omega_{(3)}^{2}\right)-\frac{k_{\infty}^{2} \mathcal{Z}_{+}}{\mathcal{Z}_{-}}\left(d z-\frac{1}{k_{\infty} \mathcal{Z}_{+}} d t\right)^{2}, \\
\hat{H}^{(1)} & =d\left(-\frac{k_{\infty}}{\mathcal{Z}_{-}} d t \wedge d z\right)+\star_{4} d \mathcal{Z}_{0}, \\
e^{-2 \hat{\phi}} & =e^{-2 \hat{\phi}_{\infty}} \mathcal{Z}_{-} / \mathcal{Z}_{0},
\end{aligned}
$$

where we have set $v=t$, to identify immediately the following 5-dimensional fields: ${ }^{17}$

$$
\begin{aligned}
d s^{2} & =\frac{1}{\mathcal{Z}_{+} \mathcal{Z}_{-}} d t^{2}-\mathcal{Z}_{0}\left(d \rho^{2}+\rho^{2} d \Omega_{(3)}^{2}\right), \\
H^{(1)} & =\star_{4} d \mathcal{Z}_{0}
\end{aligned}
$$

\footnotetext{
${ }^{16}$ The Regge slope parameter $\alpha^{\prime}$ in refs. $[17,18]$ has been replaced by $\alpha^{\prime} / 8$ here to obtain the correct form of the action and solutions.

${ }^{17}$ We have only computed $G^{(0)}$ and not $G^{(1)}$ because of its complication and because it is unnecessary to do it for the calculation of the entropy. On the other hand, the Kalb-Ramond field is customarily dualized into another vector field to which the third charge $\tilde{q}_{0}$ is associated.
} 


$$
\begin{aligned}
F & =d\left(-\frac{1}{k_{\infty} \mathcal{Z}_{+}} d t\right), \\
G^{(0)} & =d\left(-\frac{k_{\infty}}{\mathcal{Z}_{-}} d t\right), \\
e^{-2\left(\phi-\phi_{\infty}\right)} & =\sqrt{\mathcal{Z}_{+} \mathcal{Z}_{-}} / \mathcal{Z}_{0}, \\
k / k_{\infty} & =\sqrt{\mathcal{Z}_{+} / \mathcal{Z}_{-}},
\end{aligned}
$$

and the $\mathrm{T}$ duality even and odd 2-forms

$$
K^{ \pm}=-\frac{1}{\sqrt{\mathcal{Z}_{+} \mathcal{Z}_{-}}}\left(\frac{\mathcal{Z}_{+}^{\prime}}{\mathcal{Z}_{+}} \pm \frac{\mathcal{Z}_{-}^{\prime}}{\mathcal{Z}_{-}}\right) d \rho \wedge d t
$$

where a prime indicates derivative with respect to $\rho$.

In the Vielbein basis

$$
e^{0}=\frac{1}{\sqrt{\mathcal{Z}_{+} \mathcal{Z}_{-}}} d t, \quad e^{1}=\sqrt{\mathcal{Z}_{0}} d \rho, \quad e^{i}=\frac{1}{2} \sqrt{\mathcal{Z}_{0}} \rho \theta^{i},
$$

where the $\theta^{i}$ are the left-invariant $\mathrm{SU}(2)$ Maurer-Cartan 1-forms that satisfy $d \Omega^{2}{ }_{(3)}=\frac{1}{4} \theta^{i} \theta^{i}$, the binormal is given by just $\epsilon^{01}=+1$ and the entropy formula in eqs. (5.2a) and (5.2b) becomes

$$
S=\frac{1}{4 G_{N}^{(5)}} \int_{\Sigma} d^{3} x e^{-2\left(\phi-\phi_{\infty}\right)} \sqrt{|h|}\left\{1+\frac{\alpha^{\prime}}{4}\left[-2 R^{0101}+\left(K^{(-) 01}\right)^{2}+\left(K^{(+) 01}\right)^{2}\right]\right\} .
$$

The fields in the integrand are only functions of $\rho$ and we can perform the integral over $S^{3}$. Evaluating the zeroth-order term at $\rho=0$, where the horizon is located, we get

$$
\begin{aligned}
S= & \frac{1}{4 G_{N}^{(5)}}\left\{A_{\mathcal{H}}+\alpha^{\prime} \pi^{2} \lim _{\rho \rightarrow 0} \rho^{3} \sqrt{\mathcal{Z}_{0} \mathcal{Z}_{+} \mathcal{Z}_{-}}\left[-\sqrt{\frac{\mathcal{Z}_{+} \mathcal{Z}_{-}}{\mathcal{Z}_{0}}}\left[\frac{1}{\sqrt{\mathcal{Z}_{0}}}\left(\frac{1}{\sqrt{\mathcal{Z}_{+} \mathcal{Z}_{-}}}\right)^{\prime}\right]^{\prime}\right.\right. \\
& \left.\left.+\frac{1}{\mathcal{Z}_{0}}\left(\frac{\mathcal{Z}_{+}^{\prime}}{\mathcal{Z}_{+}}\right)^{2}+\frac{1}{\mathcal{Z}_{0}}\left(\frac{\mathcal{Z}_{-}^{\prime}}{\mathcal{Z}_{-}}\right)^{2}\right]\right\},
\end{aligned}
$$

where $A_{\mathcal{H}}$, the area of the horizon, is given by

$$
A_{\mathcal{H}}=2 \pi^{2} \lim _{\rho \rightarrow 0} \rho^{3} \sqrt{\mathcal{Z}_{0} \mathcal{Z}_{+} \mathcal{Z}_{-}}=2 \pi^{2} \sqrt{\tilde{q}_{0} \tilde{q}_{+} \tilde{q}_{-}} .
$$

Finally, we arrive at

$$
S=\frac{A_{\mathcal{H}}}{4 G_{N}^{(5)}}\left\{1+\frac{2 \alpha^{\prime}}{\tilde{q}_{0}}\right\}
$$

In order to compare this result with the microscopic entropy in ref. [32], we have to express the charges $\tilde{q}_{+}, \tilde{q}_{-}, \tilde{q}_{0}$ in terms of the asymptotic charges. ${ }^{18}$ First, we have to take into account the relation between $\tilde{q}_{+}, \tilde{q}_{-}, \tilde{q}_{0}$ and the numbers of fundamental strings $n$, momentum $w$ and S5-branes $N$

$$
\tilde{q}_{+}=\frac{\alpha^{\prime 2} g_{s}^{2} n}{R_{z}^{2}}, \quad \tilde{q}_{-}=\alpha^{\prime} g_{s}^{2} w, \quad \tilde{q}_{0}=\alpha^{\prime} N
$$

\footnotetext{
${ }^{18}$ See refs. [17, 33], specially eqs. $(2.18),(2.20),(2.21)$ of the later.
} 
Second, 10-dimensional Newton constant $G_{N}^{(10)}$ is given in terms of the Regge slope parameter $\alpha^{\prime}=\ell_{s}^{2}$ and the 10-dimensional string coupling constant $g_{s}$ by

$$
G_{N}^{(10)}=8 \pi^{6} g_{s}^{2} \alpha^{\prime 4}
$$

This and eq. (5.1) allow us to rewrite the entropy eq. (5.13) in the form

$$
S=2 \pi \sqrt{n w N}\left(1+\frac{2}{N}\right)
$$

Finally, in terms of the asymptotic charges $Q_{+}, Q_{-}, Q_{0}$, which are related to the numbers of branes by

$$
Q_{+}=n\left(1+\frac{2}{N}\right) \quad Q_{-}=w, \quad Q_{0}=N-1,
$$

the entropy takes the final form that can be compared with the microscopic formula

$$
S=2 \pi \sqrt{Q_{+} Q_{-}\left(Q_{0}+3\right)} .
$$

\section{Discussion}

In this paper we have performed the complete dimensional reduction of the Heterotic Superstring effective action to first order in $\alpha^{\prime}$ using the formulation based on the supersymmetry completion of the Lorentz Chern-Simons terms that occur in the Kalb-Ramond field strength $[2,3]$. We have found a $\mathbb{Z}_{2}$ transformation of the dimensionally-reduced action that leaves it invariant and that is an $\mathcal{O}\left(\alpha^{\prime}\right)$ generalization of the standard transformations that interchange KK and winding vectors and invert the KK scalar. In 10-dimensional variables (the components of the 10-dimensional fields) these transformations are nothing but the $\alpha^{\prime}$-corrected Buscher rules of the Heterotic Superstring theory, first found in [42].

Then, we used the dimensionally-reduced action to find, following the Iyer-Wald prescription $[25,26]$ an entropy formula for stringy black holes that can be obtained from a 10-dimensional solution by a single non-trivial compactification on a circle, supplemented by a trivial compactification on a torus. This formula was successfully applied to a nonextremal 4-dimensional Reissner-Nordström black hole in ref. [21] and, in this paper, we have applied it to the $\alpha^{\prime}$-corrected heterotic version of the Strominger-Vafa black hole of ref. [17] obtaining an entropy formula that matches the microscopic result obtained in [32] once the relations between integration constants and asymptotic brane charges have been correctly taken into account. As explained in ref. [33], the result obtained in ref. [17] misses a factor of 2 that we recover here.

It would be desirable to obtain an entropy formula that could be applied to more general black holes, in particular, to the 4-dimensional 4-charge ones. This requires a more general toroidal compactification of the action along the lines of the one recently obtained in ref. [9], including YM fields and closer to the Bergshoeff-de Roo formulation [58]. It is also necessary to prove the first law of black hole mechanics for the Heterotic Superstring effective action in order to make sure that the Iyer-Wald prescription can be unambiguously 
applied as we have done here or that the entropy is just given by the integral of the Noether charge as assumed in ref. [31], since, in presence of matter fields, some terms of the total Noether charge can be related to other terms in the first law [57].

Finally, let us comment on the invariance of the black-hole temperature and entropy under $\alpha^{\prime}$-corrected $\mathrm{T}$ duality, which we already mentioned in footnote 12 in the Introduction, which was discussed in refs. [31, 50, 51]. T duality manifests itself in the non-compact dimensions in which black-hole solutions appear as such as a symmetry that only acts on some lower-dimensional vector fields (KK and winding vector fields) and on some scalars (KK scalars and scalars originating in the Kalb-Ramond 2-form, if one compactifies more than one dimension). In particular, the lower-dimensional dilaton, the string metric and the lower-dimensional Kalb-Ramond field are T duality-invariant. ${ }^{19}$ Since the surface gravity is computed directly on the dimensionally-reduced metric, its invariance implies immediately the invariance of the Hawking temperature. The invariance of the lower-dimensional action and the invariance of the Riemann tensor (which follows that of the metric) automatically imply the invariance of the Wald entropy formula. In our case, the invariance of the entropy formula in eqs. (5.2a), (5.2b) is manifest.

\section{Acknowledgments}

TO would like to thank Pedro F. Ramírez and J.J. Fernández-Melgarejo for many useful conversations and Pablo A. Cano for useful comments on the manuscript. This work has been supported in part by the MCIU, AEI, FEDER (UE) grant PGC2018-095205B-I00 and by the Spanish Research Agency (Agencia Estatal de Investigación) through the grant IFT Centro de Excelencia Severo Ochoa SEV-2016-0597. The work of ZE has also received funding from "la Caixa" Foundation (ID 100010434), under the agreement LCF/BQ/DI18/11660042. TO wishes to thank M.M. Fernández for her permanent support.

\section{A Relation between 10- and 9-dimensional fields at zeroth order in $\alpha^{\prime}$}

At zeroth order in $\alpha^{\prime}$, the 10-dimensional fields can be expressed in terms of the 9dimensional ones as follows:

$$
\begin{aligned}
\hat{g}_{\mu \nu} & =g_{\mu \nu}-k^{2} A_{\mu} A_{\nu}, \\
\hat{g}_{\mu \underline{z}} & =-k^{2} A_{\mu}, \\
\hat{g}_{\underline{z z}} & =-k^{2}, \\
\hat{B}_{\mu \nu} & =B^{(0)}{ }_{\mu \nu}-A_{[\mu} B^{(0)}{ }_{\nu]}, \\
\hat{B}_{\mu \underline{z}} & =B^{(0)}{ }_{\mu}, \\
\hat{\phi} & =\phi+\frac{1}{2} \log k .
\end{aligned}
$$

\footnotetext{
${ }^{19}$ More precisely, in toroidal compactifications, the lower-dimensional Kalb-Ramond 2-form is only invariant up to a compensating $\mathrm{O}(n)$ gauge transformation [58], but its field strength is exactly invariant.
} 
The inverse relations are

$$
\begin{aligned}
g_{\mu \nu} & =\hat{g}_{\mu \nu}-\hat{g}_{\underline{z} \mu} \hat{g}_{\underline{z} \nu} / \hat{g}_{\underline{z z}}, \\
A_{\mu} & =\hat{g}_{\mu \underline{z}} / \hat{g}_{\underline{z} z}, \\
k & =\left|\hat{g}_{\underline{z z}}\right|^{1 / 2}, \\
B_{\mu \nu}^{(0)} & =\hat{B}_{\mu \nu}+\hat{g}_{\underline{z}[\mu} \hat{B}_{\nu] \underline{z}} / \hat{g}_{\underline{z z}}, \\
B^{(0)}{ }_{\mu} & =\hat{B}_{\mu \underline{z}}, \\
\phi & =\hat{\phi}-\frac{1}{4} \log \left(-\hat{g}_{\underline{z z}}\right) .
\end{aligned}
$$

\section{B Relation between 10- and 9-dimensional fields at $\mathcal{O}\left(\alpha^{\prime}\right)$}

At first order in $\alpha^{\prime}$, the 10-dimensional fields can be expressed in terms of the 9-dimensional ones as follows:

$$
\begin{aligned}
\hat{g}_{\mu \nu} & =g_{\mu \nu}-k^{2} A_{\mu} A_{\nu}, \\
\hat{g}_{\mu \underline{z}} & =-k^{2} A_{\mu}, \\
\hat{g}_{\underline{z z}} & =-k^{2}, \\
\hat{B}_{\mu \nu} & =B^{(1)}{ }_{\mu \nu}-A_{[\mu}\left[B_{\nu]}^{(1)}+\frac{\alpha^{\prime}}{2} k\left(\varphi_{A} A_{\mid \nu]}^{A}+\frac{1}{2} \Omega_{(-) \mid \nu]}^{(0)} K^{a b} K_{a b}^{(+)}-K^{(-)}{ }_{\mid \nu]}{ }^{a} \partial_{a} \log k\right)\right], \\
\hat{B}_{\mu \underline{z}} & =B^{(1)}{ }_{\mu}+\frac{\alpha^{\prime}}{4} k\left(\varphi_{A} A_{\mu}^{A}+\frac{1}{2} \Omega_{(-) \mu}^{(0)}{ }^{a b} K^{(+)}{ }_{a b}-K^{(-)}{ }_{\mu}^{a} \partial_{a} \log k\right), \\
\hat{\phi} & =\phi+\frac{1}{2} \log k, \\
\hat{A}^{A}{ }_{\mu} & =A^{A}{ }_{\mu}+k \varphi^{A} A_{\mu}, \\
\hat{A}^{A}{ }_{\underline{z}} & =k \varphi^{A} .
\end{aligned}
$$

The inverse relations are

$$
\begin{aligned}
& g_{\mu \nu}=\hat{g}_{\mu \nu}-\hat{g}_{\underline{z} \mu} \hat{g}_{\underline{z} \nu} / \hat{g}_{\underline{z z}}, \\
& A_{\mu}=\hat{g}_{\mu \underline{z}} / \hat{g}_{\underline{z z}}, \\
& k=\left|\hat{g}_{\underline{z z}}\right|^{1 / 2}, \\
& B_{\mu \nu}^{(1)}=\hat{B}_{\mu \nu}+\hat{g}_{\underline{z}[\mu}\left[\hat{B}_{\mid \nu] \underline{z}}+\frac{\alpha^{\prime}}{4}\left(\hat{A}_{\mid \nu]}^{A} \hat{A}_{A \underline{z}}+\hat{\Omega}_{(-) \mid \nu]}^{(0)} \hat{a}_{\hat{b}} \hat{\Omega}_{(-) \underline{z}}^{(0)} \hat{b}\right)\right] / \hat{g}_{\underline{z z}}, \\
& B_{\mu}^{(1)}=\hat{B}_{\mu \underline{z}}-\frac{\alpha^{\prime}}{4}\left[\hat{A}_{\mu}^{A} \hat{A}_{A \underline{z}}+\hat{\Omega}_{(-) \mu}^{(0)} \hat{a} \hat{b} \hat{\Omega}_{(-) \underline{z}}^{(0)} \hat{b} \hat{a}^{-} \hat{g}_{\mu \underline{z}}\left(\hat{A}_{\underline{z}}^{A} \hat{A}_{A \underline{z}}+\hat{\Omega}_{(-) \underline{z}}^{(0)} \hat{a}_{\hat{b}} \hat{\Omega}_{(-) \underline{z}}^{(0)} \hat{b}\right) / \hat{g}_{\underline{z z}}\right], \\
& \phi=\hat{\phi}-\frac{1}{4} \log \left(-\hat{g}_{\underline{z z}}\right), \\
& A_{\mu}^{A}=\hat{A}_{\mu}^{A}-\hat{A}_{\underline{z}}^{A} \hat{g}_{\mu \underline{z}} / \hat{g}_{\underline{z z}}, \\
& \varphi^{A}=\hat{A}_{\underline{z}}^{A} /\left(-\hat{g}_{\underline{z z}}\right)^{1 / 2} .
\end{aligned}
$$


Open Access. This article is distributed under the terms of the Creative Commons Attribution License (CC-BY 4.0), which permits any use, distribution and reproduction in any medium, provided the original author(s) and source are credited.

\section{References}

[1] A. Strominger and C. Vafa, Microscopic origin of the Bekenstein-Hawking entropy, Phys. Lett. B 379 (1996) 99 [hep-th/9601029] [INSPIRE].

[2] E.A. Bergshoeff and M. de Roo, The Quartic Effective Action of the Heterotic String and Supersymmetry, Nucl. Phys. B 328 (1989) 439 [InSPIRE].

[3] E. Bergshoeff and M. de Roo, Supersymmetric Chern-Simons Terms in Ten-dimensions, Phys. Lett. B 218 (1989) 210 [INSPIRE].

[4] C.G. Callan Jr., E.J. Martinec, M.J. Perry and D. Friedan, Strings in Background Fields, Nucl. Phys. B 262 (1985) 593 [inSPIRE].

[5] D.J. Gross and J.H. Sloan, The Quartic Effective Action for the Heterotic String, Nucl. Phys. B 291 (1987) 41 [INSPIRE].

[6] R.R. Metsaev and A.A. Tseytlin, Order $\alpha^{\prime}$ (Two Loop) Equivalence of the String Equations of Motion and the $\sigma$-model Weyl Invariance Conditions: Dependence on the Dilaton and the Antisymmetric Tensor, Nucl. Phys. B 293 (1987) 385 [INSPIRE].

[7] C.M. Hull and P.K. Townsend, The Two Loop $\beta$-function for $\sigma$ Models With Torsion, Phys. Lett. B 191 (1987) 115 [INSPIRE].

[8] W.A. Chemissany, M. de Roo and S. Panda, $\alpha^{\prime}$-Corrections to Heterotic Superstring Effective Action Revisited, JHEP 08 (2007) 037 [arXiv:0706.3636] [INSPIRE].

[9] C. Eloy, O. Hohm and H. Samtleben, Duality Invariance and Higher Derivatives, Phys. Rev. D 101 (2020) 126018 [arXiv:2004.13140] [INSPIRE].

[10] J. Maharana and J.H. Schwarz, Noncompact symmetries in string theory, Nucl. Phys. B 390 (1993) 3 [hep-th/9207016] [INSPIRE].

[11] A.H. Chamseddine, $N=4$ Supergravity Coupled to $N=4$ Matter, Nucl. Phys. B 185 (1981) 403 [INSPIRE].

[12] T. Mohaupt, Black hole entropy, special geometry and strings, Fortsch. Phys. 49 (2001) 3 [hep-th/0007195] [INSPIRE].

[13] A. Sen, Black Hole Entropy Function, Attractors and Precision Counting of Microstates, Gen. Rel. Grav. 40 (2008) 2249 [arXiv: 0708.1270] [INSPIRE].

[14] P. Dominis Prester, $\alpha^{\prime}$-Corrections and Heterotic Black Holes, in proceedings of the Black Holes in General Relativity and String Theory, Veli Lošinj, Croatia, 24-30 August 2008, arXiv:1001.1452 [INSPIRE].

[15] Y. Pang, Extended Attractor Mechanism and Non-Renormalization Theorem in 6D $(1,0)$ Supergravity, arXiv:1910.10192 [INSPIRE].

[16] D.D.K. Chow and Y. Pang, Rotating Strings in Six-Dimensional Higher-Derivative Supergravity, Phys. Rev. D 100 (2019) 106004 [arXiv: 1906. 07426] [INSPIRE].

[17] P.A. Cano, P. Meessen, T. Ortín and P.F. Ramírez, $\alpha^{\prime}$-corrected black holes in String Theory, JHEP 05 (2018) 110 [arXiv: 1803.01919] [INSPIRE]. 
[18] S. Chimento, P. Meessen, T. Ortín, P.F. Ramírez and A. Ruipérez, On a family of $\alpha^{\prime}$-corrected solutions of the Heterotic Superstring effective action, JHEP 07 (2018) 080 [arXiv: 1803.04463] [INSPIRE].

[19] P.A. Cano, S. Chimento, P. Meessen, T. Ortín, P.F. Ramírez and A. Ruipérez, Beyond the near-horizon limit: Stringy corrections to Heterotic Black Holes, JHEP 02 (2019) 192 [arXiv: 1808.03651] [inSPIRE].

[20] P.A. Cano, T. Ortín and P.F. Ramírez, On the extremality bound of stringy black holes, JHEP 02 (2020) 175 [arXiv: 1909.08530] [INSPIRE].

[21] P.A. Cano, S. Chimento, R. Linares, T. Ortín and P.F. Ramírez, $\alpha^{\prime}$ corrections of Reissner-Nordström black holes, JHEP 02 (2020) 031 [arXiv:1910.14324] [INSPIRE].

[22] P.A. Cano, P.F. Ramírez and A. Ruipérez, The small black hole illusion, JHEP 03 (2020) 115 [arXiv: 1808.10449] [INSPIRE].

[23] A. Ruipérez, Higher-derivative corrections to small black rings, arXiv:2003.02269 [INSPIRE].

[24] J. Lee and R.M. Wald, Local symmetries and constraints, J. Math. Phys. 31 (1990) 725 [INSPIRE].

[25] R.M. Wald, Black hole entropy is the Noether charge, Phys. Rev. D 48 (1993) R3427(R) [gr-qc/9307038] [INSPIRE].

[26] V. Iyer and R.M. Wald, Some properties of Noether charge and a proposal for dynamical black hole entropy, Phys. Rev. D 50 (1994) 846 [gr-qc/9403028] [InSPIRE].

[27] J.M. Bardeen, B. Carter and S.W. Hawking, The Four laws of black hole mechanics, Commun. Math. Phys. 31 (1973) 161 [InSPIRE].

[28] T. Jacobson and A. Mohd, Black hole entropy and Lorentz-diffeomorphism Noether charge, Phys. Rev. D 92 (2015) 124010 [arXiv:1507.01054] [INSPIRE].

[29] K. Prabhu, The First Law of Black Hole Mechanics for Fields with Internal Gauge Freedom, Class. Quant. Grav. 34 (2017) 035011 [arXiv:1511.00388] [INSPIRE].

[30] P.B. Aneesh, S. Chakraborty, S.J. Hoque and A. Virmani, First law of black hole mechanics with fermions, Class. Quant. Grav. 37 (2020) 205014 [arXiv:2004.10215] [INSPIRE].

[31] J.D. Edelstein, K. Sfetsos, J.A. Sierra-García and A. Vilar López, T-duality equivalences beyond string theory, JHEP 05 (2019) 082 [arXiv:1903.05554] [INSPIRE].

[32] P. Kraus and F. Larsen, Holographic gravitational anomalies, JHEP 01 (2006) 022 [hep-th/0508218] [INSPIRE].

[33] F. Faedo and P.F. Ramírez, Exact charges from heterotic black holes, JHEP 10 (2019) 033 [arXiv: 1906.12287] [INSPIRE].

[34] T. Ortín, work in progress.

[35] R.R. Khuri and T. Ortín, A Nonsupersymmetric dyonic extreme Reissner-Nordstrom black hole, Phys. Lett. B $\mathbf{3 7 3}$ (1996) 56 [hep-th/9512178] [INSPIRE].

[36] E. Álvarez, L. Álvarez-Gaumé and Y. Lozano, An Introduction to T duality in string theory, Nucl. Phys. B Proc. Suppl. 41 (1995) 1 [hep-th/9410237] [inSPIRE].

[37] T.H. Buscher, A Symmetry of the String Background Field Equations, Phys. Lett. B 194 (1987) 59 [INSPIRE].

[38] T.H. Buscher, Path Integral Derivation of Quantum Duality in Nonlinear $\sigma$-models, Phys. Lett. B 201 (1988) 466 [INSPIRE]. 
[39] E. Bergshoeff, I. Entrop and R. Kallosh, Exact duality in string effective action, Phys. Rev. D 49 (1994) 6663 [hep-th/9401025] [INSPIRE].

[40] E. Bergshoeff, C.M. Hull and T. Ortín, Duality in the type-II superstring effective action, Nucl. Phys. B 451 (1995) 547 [hep-th/9504081] [INSPIRE].

[41] P. Meessen and T. Ortín, An $\mathrm{SL}(2, \mathbb{Z})$ multiplet of nine-dimensional type-II supergravity theories, Nucl. Phys. B 541 (1999) 195 [hep-th/9806120] [INSPIRE].

[42] E. Bergshoeff, B. Janssen and T. Ortín, Solution generating transformations and the string effective action, Class. Quant. Grav. 13 (1996) 321 [hep-th/9506156] [INSPIRE].

[43] M. Serone and M. Trapletti, A Note on T-duality in heterotic string theory, Phys. Lett. B 637 (2006) 331 [hep-th/0512272] [INSPIRE].

[44] O.A. Bedoya, D. Marqués and C. Núñez, Heterotic $\alpha^{\prime}$-corrections in Double Field Theory, JHEP 12 (2014) 074 [arXiv: 1407.0365] [INSPIRE].

[45] K.A. Meissner, Symmetries of higher order string gravity actions, Phys. Lett. B 392 (1997) 298 [hep-th/9610131] [INSPIRE].

[46] N. Kaloper and K.A. Meissner, Duality beyond the first loop, Phys. Rev. D 56 (1997) 7940 [hep-th/9705193] [INSPIRE].

[47] O. Hohm and B. Zwiebach, Green-Schwarz mechanism and $\alpha^{\prime}$-deformed Courant brackets, JHEP 01 (2015) 012 [arXiv: 1407.0708] [INSPIRE].

[48] D. Marqués and C.A. Núñez, T-duality and $\alpha^{\prime}$-corrections, JHEP 10 (2015) 084 [arXiv: 1507.00652] [INSPIRE].

[49] W.H. Baron, J.J. Fernández-Melgarejo, D. Marqués and C. Núñez, The Odd story of $\alpha^{\prime}$-corrections, JHEP 04 (2017) 078 [arXiv: 1702.05489] [INSPIRE].

[50] G.T. Horowitz and D.L. Welch, Duality invariance of the Hawking temperature and entropy, Phys. Rev. D 49 (1994) 590 [hep-th/9308077] [INSPIRE].

[51] J.D. Edelstein, K. Sfetsos, J.A. Sierra-García and A. Vilar López, T-duality and high-derivative gravity theories: the BTZ black hole/string paradigm, JHEP 06 (2018) 142 [arXiv: 1803.04517] [INSPIRE].

[52] T. Ortín, Gravity and Strings, 2nd edition, in Cambridge Monographs on Mathematical Physics, Cambridge University Press, Cambridge U.K. (2015).

[53] A. Fontanella and T. Ortín, On the supersymmetric solutions of the Heterotic Superstring effective action, JHEP 06 (2020) 106 [arXiv: 1910.08496] [INSPIRE].

[54] J. Scherk and J.H. Schwarz, How to Get Masses from Extra Dimensions, Nucl. Phys. B 153 (1979) 61 [INSPIRE].

[55] E. Bergshoeff, M. de Roo, B. de Wit and P. van Nieuwenhuizen, Ten-Dimensional Maxwell-Einstein Supergravity, Its Currents, and the Issue of Its Auxiliary Fields, Nucl. Phys. B 195 (1982) 97 [INSPIRE].

[56] G.F. Chapline and N.S. Manton, Unification of Yang-Mills Theory and Supergravity in Ten-Dimensions, Phys. Lett. B 120 (1983) 105 [INSPIRE].

[57] G. Compère, Note on the First Law with p-form potentials, Phys. Rev. D 75 (2007) 124020 [hep-th/0703004] [inSPIRE].

[58] T. Ortín, $O(n, n)$ invariance and Wald entropy formula in the Heterotic Superstring effective action at first order in $\alpha^{\prime}$, arXiv:2005.14618 [INSPIRE]. 